\title{
Synthesis and Spectroscopic and Biological Activities of Zn(II) Porphyrin with Oxygen Donors
}

\author{
Gauri Devi Bajju, ${ }^{1}$ Sujata Kundan, ${ }^{1}$ Madhulika Bhagat, ${ }^{2}$ Deepmala Gupta, \\ Ashu Kapahi, ${ }^{1}$ and Geeta Devi ${ }^{1}$ \\ ${ }^{1}$ Department of Chemistry, University of Jammu, New Campus, Baba Sahib Ambedkar Road, Jammu, \\ Jammu and Kashmir 180 006, India \\ ${ }^{2}$ School of Biotechnology, University of Jammu, New Campus, Baba Sahib Ambedkar Road, Jammu, \\ Jammu and Kashmir 180 006, India
}

Correspondence should be addressed to Gauri Devi Bajju; gauribajju@gmail.com

Received 16 October 2013; Accepted 4 December 2013; Published 16 March 2014

Academic Editor: Ian Butler

Copyright (C) 2014 Gauri Devi Bajju et al. This is an open access article distributed under the Creative Commons Attribution License, which permits unrestricted use, distribution, and reproduction in any medium, provided the original work is properly cited.

Results of investigation of the physicochemical properties of zinc complexes containing substituted phenols as axial ligand having general formula $\left[\mathrm{X}-\mathrm{Zn}-\mathrm{t}\left(p-\mathrm{CH}_{3}\right) \mathrm{PP}\right.$ ] [where $\mathrm{X}=$ different phenolates as axial ligand] in impurity-free organic solvent are presented. The four-coordinated zinc porphyrin accepts one axial ligand in 1:1 molar ratio to form five-coordinated complex, which is purified by column chromatography and characterized by physicochemical, biological evaluation and TGA/DTA studies. Absorption spectra show two principal effects: a red shift for phenols bearing substituted electron releasing groups $\left(-\mathrm{CH}_{3},-\mathrm{NH}_{2}\right)$ and blue shift for phenols bearing electron withdrawing groups $\left(-\mathrm{NO}_{2},-\mathrm{Cl}\right)$ relative to $\mathrm{Zn}-\mathrm{t}\left(p-\mathrm{CH}_{3}\right)$ PP, respectively. ${ }^{1} \mathrm{H} \mathrm{NMR}$ spectra show that the protons of the phenol ring axially attached to the central metal ion are merged with the protons of the porphyrin ring. Fluorescence spectra show two fluorescence peaks in the red region with emission ranging from $550 \mathrm{~nm}$ to $700 \mathrm{~nm}$. IR spectra confirm the appearance of $\mathrm{Zn}-\mathrm{N}_{\mathrm{Por}}$ and $\mathrm{Zn}-\mathrm{O}$ vibrational frequencies, respectively. According to the thermal studies, the complexes have a higher thermal stability and the decomposition temperature of these complexes depends on the axial ligation. The respective complexes of $\mathrm{X}-\mathrm{Zn}^{\mathrm{II}}-\mathrm{t}\left(p-\mathrm{CH}_{3}\right) \mathrm{PP}$ were found to possess higher antifungal activity (up to $90 \%$ ) and higher in vitro cytotoxicity against human cancer cells lines.

\section{Introduction}

The involvement of porphyrins in many biological processes and the possibility of tailoring their physical and chemical properties at the molecular level make the porphyrins and metalloporphyrins versatile synthetic base material for research areas due to their immense biological and fascinating importance in many technical applications including, but not limited to, sensors, solar cells, as catalysts [1-6], optical monomers [7, 8], photocatalysts [9-12], photosensitizers in photodynamic therapy (PDT) [13], supramolecular chemistry [14, 15], ionophores [16], and for the treatment of tumors and malignant tissues [17] in combination with electromagnetic radiation or radioactive emissions, as photosensitizers for dye sensitized solar cells (DSSCs) $[18,19]$. They are also regarded as nature's choice catalysts and carry out a remarkable spectrum of bioenergetics reaction ranging from photosynthetic energy transduction to generation of ammonia, regiospecific oxygen transfer (hydroxylation and epoxidation), and conversion of carbon dioxide to hydrocarbons. The free base 5,10,15,20-meso-tetraphenylporphyrin $\left(\mathrm{H}_{2} \mathrm{TPP}\right)$ and the meso-substituted-tetra(ortho- and para-phenyl) porphyrin derivatives $\left(-\mathrm{CH}_{3},-\mathrm{OCH}_{3},-\mathrm{Cl}\right.$, or $\left.-\mathrm{NEt}_{2}\right)$ have been reviewed in literature and were synthesized according to the documented procedure [20]. Over the past decades, many examples of axial coordination properties of metalloporphyrins with $\mathrm{S}, \mathrm{O}, \mathrm{P}$, and $\mathrm{N}$ bases have been reported $[21,22]$. The interaction of metalloporphyrins with donor molecules via axial coordination either in their ground and excited state can strongly influence the absorption properties and 
the efficiency of energy or electron transfer processes [23, 24]. This ability of metalloporphyrins to attach additional ligand (extra coordination) determines their role in enzyme and catalytic processes. Mono- or bidentate complexes form, depending on the system of substituent in the porphyrin macrocycle, central ion, or the nature and concentration of the extra ligand. The reactions of extra coordination attract attention on both theoretical and experimental researches [25]. Also, the detailed studies on the solvation and axial ligation properties of ZnTPP have been reported [26, 27]. These investigations have shed light on how axial ligands induce changes in the spectral absorption features and the electrochemistry of metalloporphyrins. The ability for numerous chemical modifications and the large number of different mechanisms by which porphyrins affect microbial and viral pathogens place porphyrins into a group of compounds with an outstanding potential for discovery of novel agents, procedures, and materials active against pathogenic microorganisms [28]. Metalloporphyrins are the basis of new antifungal, antiparasitic, and anticancer drugs because modification of the porphyrin periphery confers qualitatively a new spectrum of activities to metalloporphyrins [29, 30]. Metal complexes are well known to accelerate drug action and the efficiency of a therapeutic agent can often be enhanced upon coordination with a metal ion [31]. Of particular interest metal ions such as zinc, which is a natural component of insulin required for the regulation of sugar metabolism and it is also incorporated into the catalytic proteins to act as a metalloenzyme that facilitate a multitude of chemical reactions needed for life. $\mathrm{Zn}$ (II) metal complexes with different ligands show overall good potential for antibacterial, antifungal, antioxidant, and anticancer activities [32, 33]. Zinc also forms low molecular weight complexes and, therefore, proves to be more beneficial against several diseases. Various biological aspects of the metal-based drugs/ligands entirely depend on the ease of cleaving the bond between the metal ion and the ligand. As a consequence, it is essential to understand the relationship between ligand and the metal in biological systems. With this objective, we aimed at synthesis, spectroscopic characterization, and biological studies of axial zinc(II)-5,10,15,20-mesotetra(para-methylphenyl)porphyrins with different phenols as axial ligand.

\section{Experimental}

2.1. Materials and Instruments. All the chemicals were of analytical grade and used as received unless otherwise noted. Pyrrole (Fluka, Switzerland) was distilled at room temperature over potassium hydroxide $(\mathrm{KOH})$ pellets under reduced pressure before use. $p$-Tolualdehyde ( $p$-methylbenzaldehyde) (Aldrich, USA), propionic acid (Qualigens, India), silica gel $(60-120 \mathrm{mesh})$ and silica gel (TLC grade, particle size = $75 \mu$ (Merck, Germany), aluminum oxide (basic) for column chromatography (Fluka, Switzerland), and zinc acetate $\left(\mathrm{Zn}(\mathrm{OAc})_{2} \cdot 2 \mathrm{H}_{2} \mathrm{O}\right)$ (E. Merck, India) were used as supplied. Organic solvents were degassed by purging with prepurified nitrogen gas and dried before use. The various phenols used were of AR grade (Sisco Research Laboratories Pvt. Ltd.) and used without further purification.
The optical absorption spectra of the compounds were recorded on a Hitachi U-3400, Lambda 35 UV-Vis spectrophotometer and Elico spectral treats UV-Vis spectrometer using a pair of matched quartz cells of $10 \mathrm{~mm}$ path length at an ambient temperature. The oscillator strength $(f)$ of the transitions in absorption spectra was calculated from the expression [34]

$$
f=4.33 \times 10^{-9} \varepsilon \Delta v_{1 / 2},
$$

where $\varepsilon$ is the molar absorption coefficient in $\mathrm{dm}^{3} \mathrm{~mol}^{-1} \mathrm{~cm}^{-1}$ and $\Delta v_{1 / 2}$ is the full width at half maximum in $\mathrm{cm}^{-1}$. IR spectra of complexes over the region $4000-400 \mathrm{~cm}^{-1}$ were recorded on PERKIN ELMER $580 \mathrm{~B}$ spectrophotometer at room temperature using $\mathrm{KBr}$ discs or Nujol mulls which confirms the $(\mathrm{M}-\mathrm{N})$ and $(\mathrm{M}-\mathrm{L})$ vibration $[35,36]$. The ${ }^{1} \mathrm{H}$ NMR spectra were recorded on a Bruker Avance II 400 $(\mathrm{MHz}) \mathrm{NMR}$ spectrometer in $\mathrm{CDCl}_{3}$ using tetramethylsilane (TMS) as internal standard. Porphyrin solutions $(0.5 \mathrm{~mL})$ of $10^{-2}$ to $10^{-3} \mathrm{~m}$ in $\mathrm{CDCl}_{3}$ were used for ${ }^{1} \mathrm{H}$ NMR studies. Carbon, hydrogen, and nitrogen were analyzed microanalytically using CHNS analyzer Leco model 932, USA, at a temperature of about $1000^{\circ} \mathrm{C}$ using helium as carrier gas and oxygen for combustion. The MALDI mass spectra were recorded in the electron-impact mode on a Finnigan 3300 spectrometer using chloroform or methanol as solvent. The steady state fluorescence measurements were performed on synergy MX Biotek Multimode reader using a quartz cell of $1 \mathrm{~cm}$ path length at ambient temperature. The thermogravimetric analyses (TGA) and differential thermal analyses (DTA) were performed on a Linseis STA PT-1000 in air atmosphere at a heating rate of $10^{\circ} \mathrm{C} / \mathrm{min}$. The right angle detection was employed for monitoring the fluorescence.

\subsection{Biological Studies}

2.2.1. Antifungal Studies. The in vitro antifungal activity has been done by disc diffusion method (DDM) against the pathogen and in vitro cytotoxicity against human cancer cell lines. In vitro antifungal activity of some of the investigated compounds was tested by agar plate technique against the pathogen "Sclerotium rolfsi" by the poisoned food method using potato dextrose agar (PDA) (glucose $20 \mathrm{~g}$, starch $20 \mathrm{~g}$, and agar-agar $20 \mathrm{~g}$ in $1000 \mathrm{~mL}$ distilled water) nutrient as the medium. Solution of the test compounds in DMSO (100 ppm, $200 \mathrm{ppm}$, and $300 \mathrm{ppm}$ concentrations) was prepared and mixed with the PDA. The medium was then poured into sterilized Petri plates and the spores of fungi were placed on the medium with the help of inoculum's needle inside laminar flow. The plates were inoculated with seven-day-old culture of the pathogen by placing $2 \mathrm{~mm}$ bit of the compound under investigation with different concentration in the centre of plates. The inoculated plates were incubated at $27^{\circ} \mathrm{C}$ for 5 days. The linear growth of fungus in control and treatment was recorded at different concentrations of the complexes after 5 days. The growth of "Sclerotium rolfsi" over control was calculated as per Vincent [37]:

$$
\% \text { inhibition }(I)=\frac{(C-T)}{C} \times 100 \text {, }
$$


where $I$ = percent inhibition, $C=$ mean growth of fungus in $(\mathrm{mm})$ in control, and $T=$ mean growth of fungus in $(\mathrm{mm})$ in treatment.

\subsubsection{In Vitro Cytotoxicity against Human Cancer Cell Lines}

Cell Lines and Cell Cultures. The human cancer lines were obtained either from National Center for Cell Science, Pune, India, or IIIM, Jammu, J\&K, India. The human prostate (PC-3), lung (A-549), and acute lymphoblastic leukemia (THP-1) cell line was grown and maintained in RPMI-1640 medium, $\mathrm{pH}$ 7.4, whereas DMEM was used for Breast (MCF7). The media were supplemented with FCS (10\%), penicillin (100 units $/ \mathrm{mL})$, streptomycin $(100 \mu \mathrm{g} / \mathrm{mL})$, and glutamine $(2 \mathrm{mM})$ and cells were grown in $\mathrm{CO}_{2}$ incubator (Heraeus, $\mathrm{GmbH}$, Germany) at $37^{\circ} \mathrm{C}$ with $90 \%$ humidity and $5 \% \mathrm{CO}_{2}$. Cells were treated with samples dissolved in DMSO while the untreated control cultures received only the vehicle (DMSO, $<0.2 \%)$.

Cytotoxicity Assay. In vitro cytotoxicity against human cancer cell lines was determined using sulphorhodamine B dye assay $[38,39]$. Both test samples stock solutions were prepared in DMSO and serially diluted with growth medium to obtain desired concentrations.

\subsection{Synthesis of Axially Ligated Zn(II) Porphyrins Complexes}

2.3.1. Synthesis of 5,10,15,20-Meso-tetra(p-methylphenyl)porphyrin $\left[\mathrm{H}_{2} t\left(p-\mathrm{CH}_{3}\right) P P\right]$. The preparation of $\mathrm{H}_{2}-\mathrm{t}\left(p-\mathrm{CH}_{3}\right) \mathrm{PP}$ was carried out by condensation of pyrrole with $p$ tolualdehyde in refluxing propionic acid, $\mathrm{H}_{2}-\mathrm{t}\left(p-\mathrm{CH}_{3}\right) \mathrm{PP}$ purified by column chromatography using chloroform as eluent (Scheme 1(a)). A second moving band was collected after evaporation of the solvent furnished violet colour as a title compound. UV-Visible $\left(\lambda_{\mathrm{nm}}(\mathrm{nm})\left(\mathrm{CHCl}_{3}\right)\right): 430$, 516, 553, 592, 649; ${ }^{1} \mathrm{H}$ NMR $\left(\mathrm{CDCl}_{3}\right):-2.77$ (s) (Imino-H), $8.86\left(\mathrm{~s}, 8 \mathrm{H}, \beta_{\mathrm{py}}-\mathrm{H}\right)$, meso-aryl protons $8.11\left(\mathrm{~d}, 8 \mathrm{H}, \mathrm{H}_{o}\right), 7.56$ $\left(\mathrm{d}, 8 \mathrm{H}, \mathrm{H}_{m}\right), 2.64\left(\mathrm{~s}, 12 \mathrm{H}, \mathrm{H}_{\mathrm{me}}\right)$; IR spectra (in $\left.\mathrm{KBr}\right)\left(\mathrm{cm}^{-1}\right)$ : imino $\nu(\mathrm{N}-\mathrm{H})$ at 3446 , aromatic $\nu(\mathrm{C}-\mathrm{H})$ at $2964, \nu(\mathrm{C}-\mathrm{N})$ at $1350, v(\mathrm{C}=\mathrm{C})$ at $1650, v(\mathrm{C}=\mathrm{N})$ at $1589, v\left(\mathrm{CH}_{3}\right)$ at 2855 ; Anal.Calcd. for $\mathrm{C}_{48} \mathrm{H}_{38} \mathrm{~N}_{4}(\%)$ : C, 86.04; H, 5.71; N, 8.6. Found: C, 86.11; H, 5.89; N, 8.42.

\subsubsection{Synthesis of Zinc(II)5,10,15,20-Meso-tetra (p-methyl-} phenyl)porphyrin $\left[\mathrm{Zn}^{I I}-\mathrm{t}\left(\mathrm{p}-\mathrm{CH}_{3}\right) \mathrm{PP}\right] . \mathrm{H}_{2}-\mathrm{t}\left(p-\mathrm{CH}_{3}\right) \mathrm{PP}(20 \mathrm{mg}$, $0.030 \mathrm{mmol})$ in chloroform $(20 \mathrm{~mL})$ and $\mathrm{Zn}(\mathrm{OAc})_{2} \cdot 2 \mathrm{H}_{2} \mathrm{O}$ $(20 \mathrm{mg}, 0.091 \mathrm{mmol})$ in methanol $(10 \mathrm{~mL})$ were refluxed for $2 \mathrm{hrs}$ at $60-70^{\circ} \mathrm{C}$ till the colour of the solution changed from purple to red. After cooling to room temperature the solvent was removed under reduced pressure and the solid residue was repeatedly washed with water $(3 \times 60 \mathrm{~mL})$ to remove the excess of zinc acetate. The filtered product was dried over anhydrous sodium sulphate and purified by column chromatography using $\left(\mathrm{Al}_{2} \mathrm{O}_{3}\right)$ as stationary phase and $\mathrm{CHCl}_{3}$ as eluent. Yield of the complex $\left[\mathrm{Zn}^{\mathrm{II}}-\mathrm{t}\left(p-\mathrm{CH}_{3}\right) \mathrm{PP}\right]$ (18 mg, 80\%) (Scheme 1(b)).

UV-Vis $\left(\lambda_{\mathrm{nm}}(\mathrm{nm})\left(\mathrm{CHCl}_{3}\right)\right): 432,564.2,609.3 ;{ }^{1} \mathrm{H}$ NMR $\left(\mathrm{CDCl}_{3}\right): 8.65\left(\mathrm{~s}, 8 \mathrm{H}, \beta_{\mathrm{py}}-\mathrm{H}\right), 7.75\left(\mathrm{~d}, 8 \mathrm{H}, \mathrm{H}_{m}\right), 8.06\left(\mathrm{~d}, 8 \mathrm{H}, \mathrm{H}_{o}\right)$, $2.69\left(\mathrm{~s}, 12 \mathrm{H},-\mathrm{CH}_{3}\right)$; IR spectra (inKBr) $\left(\mathrm{cm}^{-1}\right)$ : aromatic $\nu(\mathrm{C}-$ $\mathrm{H})$ appears at $2963, v(\mathrm{C}-\mathrm{N})$ at $1349, v(\mathrm{C}=\mathrm{C})$ at $1658, v(\mathrm{C}=\mathrm{N})$ at 1594, $v\left(\mathrm{CH}_{3}\right)$ at $2849, v(\mathrm{Zn}-\mathrm{N})$ at 482 ; Anal.Calcd. for $\mathrm{C}_{48} \mathrm{H}_{36} \mathrm{~N}_{4} \mathrm{Zn}_{1}(\%): \mathrm{C}, 78.39 ; \mathrm{H}, 4.93 ; \mathrm{N}, 7.61$. Found: C, 78.46; $\mathrm{H}, 4.99 ; \mathrm{N}, 7.72$.

2.3.3. Synthesis of $5,10,15,20$-Meso-tetra (p-methylphenyl)porphinatozinc(II)-phenoxide $\left[\mathrm{X}-\mathrm{Zn}^{I I}-t\left(p-\mathrm{CH}_{3}\right) P P\right]$. It is as follows:

$$
\mathrm{Zn}(\mathrm{II})-\mathrm{t}\left(p-\mathrm{CH}_{3}\right) \mathrm{PP}+\mathrm{X} \leftrightarrows \mathrm{X}-\mathrm{Zn}(\mathrm{II})-\mathrm{t}\left(p-\mathrm{CH}_{3}\right) \mathrm{PP}
$$

Phenol (X) $\left(3.086 \times 10^{-2}\right.$ moles $)$ in methanol $(10 \mathrm{~mL})$ and $\mathrm{Zn}-\mathrm{t}\left(p-\mathrm{CH}_{3}\right) \mathrm{PP}\left(6.602 \times 10^{-4}\right.$ moles; $\left.0.59 \mathrm{~g}\right)$ in chloroform $(15 \mathrm{~mL})$ were stirred without heating for two hours. After completion of reaction as indicated by TLC, the reaction mixture was extracted with $2 \mathrm{~N} \mathrm{NaOH}$ solution and chloroform as an eluent. The compound recovered after extraction was passed through anhydrous $\mathrm{Na}_{2} \mathrm{SO}_{4}$. The solvent was recovered under reduced pressure and chromatographed through basic alumina using chloroform as an eluent, recrystallised with petroleum ether, and characterized by UV-Vis and ${ }^{1} \mathrm{H}$ NMR spectra (Scheme 1(c)).

\section{Results and Discussion}

3.1. Synthesis and Characterization. The physical measurements and analytical data of all complexes with general formula $\left[\mathrm{X}-\mathrm{Zn}^{\mathrm{II}}-\left(p-\mathrm{CH}_{3}\right) \mathrm{PP}\right]$ ( where $\mathrm{X}=$ different phenolates as axial ligand) are shown in Tables 1-6. All the complexes are coloured, photosensitive to light, and soluble in polar solvents but water insoluble. The data showing growth inhibition of the fungus is given in Table 7 .

3.1.1. ${ }^{1} H$ NMR Spectroscopy. The ${ }^{1} \mathrm{H}$ NMR spectrum of the meso-tetra( $p$-methylphenyl)porphyrin and its $\mathrm{Zn}$ (II) derivatives containing different phenols as axial ligand was recorded in deuterated chloroform at $298 \mathrm{~K}$ (Table 1). The spectrum show a singlet at $-2.79 \mathrm{ppm}$ for inner imino protons of the $\mathrm{H}_{2} \mathrm{TPP}$, while those of $\mathrm{H}_{2}-\mathrm{t}\left(p-\mathrm{CH}_{3}\right) \mathrm{PP}$ resonate at $-2.77 \mathrm{ppm}$. The meso-aryl protons of $\mathrm{H}_{2}$ TPP resonate as a singlet at $8.19 \mathrm{ppm}$ for ortho and $7.59 \mathrm{ppm}$ for meta and para protons, respectively, but in case of $\mathrm{H}_{2}-\mathrm{t}\left(p-\mathrm{CH}_{3}\right) \mathrm{PP}$, the resonance occurs at $8.11 \mathrm{ppm}$ for ortho and $7.56 \mathrm{ppm}$ for meta protons; that is, resonance is shifted upfield relative to $\mathrm{H}_{2}$ TPP. The methyl protons of the substituted $-\mathrm{CH}_{3}$ group at the paraposition of the meso-aryl ring resonate as a singlet at $2.64 \mathrm{ppm}$. This effect of meso-substitution on the $\beta$-pyrrole protons and meso-aryl protons has earlier been reviewed in literature [40]. Further, in axially ligated zinc(II) complexes of $\mathrm{H}_{2}-\mathrm{t}\left(p-\mathrm{CH}_{3}\right) \mathrm{PP}$, a slight difference in the proton resonance is observed depending upon the nature of the axial ligand coordinated via zinc atom. In the case of $p-\mathrm{NO}_{2} \mathrm{phO}-\mathrm{Zn}$ $\mathrm{t}\left(p-\mathrm{CH}_{3}\right) \mathrm{PP}$, Figure 1 indicates that the $\beta$-pyrrole protons resonate as a singlet at $9.01 \mathrm{ppm}$ and the meso-aryl ortho protons resonate as doublet at $8.39 \mathrm{ppm}$ and $7.92 \mathrm{ppm}$ for meso-aryl meta and para protons, respectively, which are slightly downfield (deshielded) compared to $\mathrm{Zn}-\mathrm{t}\left(p-\mathrm{CH}_{3}\right) \mathrm{PP}$ 


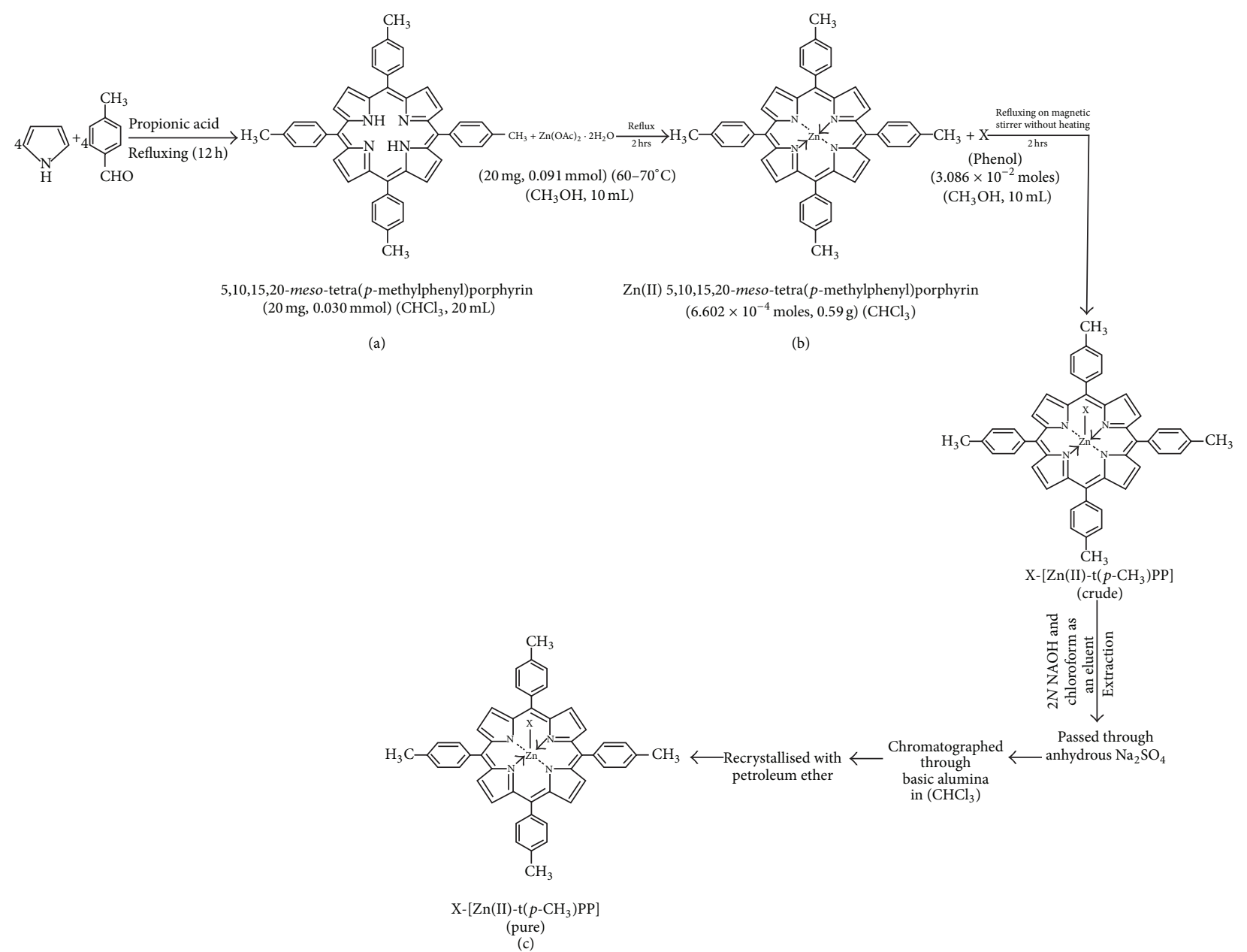

$$
\begin{aligned}
& -\mathrm{Oph} \\
& \alpha \text {-Naphthol } \\
\mathrm{X} \text { (axial ligands) }= & o-, m-\text {, and } p-\mathrm{NH}_{2} \mathrm{Oph} \\
& o-, m-\text {, and } p-\mathrm{OCH}_{3} \mathrm{Oph} \\
& o-, m-\text {, and } p-\mathrm{NO}_{2} \mathrm{Oph} \\
& o-, m-\text {, and } p-\mathrm{ClOph} \\
& \text { 2,4-Dichlorophenol }
\end{aligned}
$$

SCHEme 1: General synthetic route for the synthesis of zinc(II)-5,10,15,20-meso-tetra(para-methylphenyl)porphyrin containing different phenols as axial ligand.

as well as for $\mathrm{H}_{2}-\mathrm{t}\left(p-\mathrm{CH}_{3}\right) \mathrm{PP}$. The methyl protons of the mesoaryl rings resonate at $2.65 \mathrm{ppm}$. But in case of $p-\mathrm{OCH}_{3} \mathrm{phO}-$ $\mathrm{Zn}$ - $\mathrm{t}\left(p-\mathrm{CH}_{3}\right) \mathrm{PP}$ (Figure 2), the $\beta$-pyrrole protons resonate as a singlet at $8.93 \mathrm{ppm}$ and the meso-aryl ortho protons resonate as doublet at $8.22 \mathrm{ppm}$ and $7.66 \mathrm{ppm}$ for meta and para, respectively, which are slightly upfield (shielded) compared to $\mathrm{Zn}-\mathrm{t}\left(p-\mathrm{CH}_{3}\right) \mathrm{PP}$ as well as for $\mathrm{H}_{2}-\mathrm{t}\left(p-\mathrm{CH}_{3}\right) \mathrm{PP}$. The methyl protons of the meso-aryl rings resonate at $2.31 \mathrm{ppm}$ and the methoxy protons of para-methoxy phenolate resonate as singlet at $3.36 \mathrm{ppm}$. The ${ }^{1} \mathrm{H}$ NMR data of various axially ligated $\mathrm{Zn}(\mathrm{II})$ complexes of $\mathrm{H}_{2} \mathrm{t}\left(p-\mathrm{CH}_{3}\right) \mathrm{PP}$ revealed that phenols with electron-withdrawing groups like $-\mathrm{NO}_{2},-\mathrm{Cl}$ caused slight downfield shift (deshielding) and those with electron releasing group like $-\mathrm{CH}_{3},-\mathrm{OCH}_{3}$, and $-\mathrm{NH}_{2}$ caused upfield shift (shielding) of protons with respect to $\mathrm{Zn}$ $\mathrm{t}\left(p-\mathrm{CH}_{3}\right) \mathrm{PP}$ and $\mathrm{H}_{2} \mathrm{t}\left(p-\mathrm{CH}_{3}\right) \mathrm{PP}$ complexes.
3.1.2. Absorption Spectroscopy. The electronic spectra of a typical porphyrin contain one intense band in the nearultraviolet region of the spectrum around $400 \mathrm{~nm}$ (the soret band or B-band) depending on whether the porphyrin is $\beta$ or meso-substituted with $\varepsilon>2 \times 10^{5}$, followed by four lowintensity absorption bands at $514 \mathrm{~nm}, 550 \mathrm{~nm}, 591 \mathrm{~nm}$, and $647 \mathrm{~nm}$ (the Q-band), that is, $\mathrm{Q}_{\mathrm{y}}(1,0), \mathrm{Q}_{\mathrm{y}}(0,0), \mathrm{Q}_{\mathrm{x}}(1,0)$, and $\mathrm{Q}_{\mathrm{x}}(0,0)$, respectively. The $\mathrm{B}$ - and Q-bands both arise from $\pi \rightarrow \pi^{*}$ transition and can be explained by four frontier orbitals (HOMO and LUMO orbitals) (the Gouterman four orbital model). Ongoing from porphyrin to metalloporphyrin, the ring symmetry of the planar macrocycle fragment increases to $\mathrm{D}_{4 \mathrm{~h}}$ from $\mathrm{D}_{2 \mathrm{~h}}$ due to which the spectrum is simplified. The optical absorption data $\mathrm{Zn}(\mathrm{II})-5,10,15,20$ meso-tetra( $p$-methylphenyl)porphyrin containing different phenols as axial ligand in chloroform is listed in (Table 2). 
TABLE 1: ${ }^{1} \mathrm{H}$ NMR data ${ }^{\mathrm{a}}$ of free base $\mathrm{H}_{2}-\mathrm{t}\left(p-\mathrm{CH}_{3}\right) \mathrm{PP}$ and axially ligated $\mathrm{X}-\mathrm{Zn}-\mathrm{t}\left(p-\mathrm{CH}_{3}\right) \mathrm{PP}(\mathrm{X}=$ different phenols as axial ligand $)$ in $\mathrm{CDCl}{ }_{3}$ at $298 \mathrm{~K}$.

\begin{tabular}{|c|c|c|c|c|}
\hline Porphyrins & $\begin{array}{l}\beta \text {-Pyrrole } \\
\text { protons }\end{array}$ & $\begin{array}{l}\text { Imino } \\
\text { protons }\end{array}$ & Meso-aryl protons & Other protons \\
\hline $\begin{array}{l}\mathrm{H}_{2} \mathrm{t}\left(p-\mathrm{CH}_{3}\right) \mathrm{PP} \\
{\left[\left(\mathrm{C}_{48} \mathrm{H}_{38} \mathrm{~N}_{4}\right)\right]}\end{array}$ & $8.86(\mathrm{~s})$ & $-2.77(\mathrm{~s})$ & $\begin{array}{l}8.11\left(\mathrm{~d}, 8 \mathrm{H}, \mathrm{H}_{o}\right) \\
7.56\left(\mathrm{~d}, 8 \mathrm{H}, \mathrm{H}_{m}\right)\end{array}$ & $2.64\left(\mathrm{~s}, 12 \mathrm{H}, \mathrm{H}_{\mathrm{me}}\right)$ \\
\hline $\begin{array}{l}\mathrm{Zn}-\mathrm{t}\left(p-\mathrm{CH}_{3}\right) \mathrm{PP} \\
{\left[\left(\mathrm{C}_{48} \mathrm{H}_{36} \mathrm{~N}_{4} \mathrm{Zn}_{1}\right)\right]}\end{array}$ & $8.65(\mathrm{~s})$ & - & $\begin{array}{l}8.06\left(\mathrm{~d}, 8 \mathrm{H}, \mathrm{H}_{o}\right) \\
7.75\left(\mathrm{~m}, 8 \mathrm{H}, \mathrm{H}_{m}\right)\end{array}$ & $2.69\left(\mathrm{~s}, 12 \mathrm{H}, \mathrm{H}_{\mathrm{me}}\right)$ \\
\hline $\begin{array}{l}\mathrm{phO}-\mathrm{Zn}-\mathrm{t}\left(p-\mathrm{CH}_{3}\right) \mathrm{PP} \\
{\left[\left(\mathrm{C}_{6} \mathrm{H}_{5} \mathrm{O}\right) \mathrm{Zn}\left(\mathrm{C}_{48} \mathrm{H}_{36} \mathrm{~N}_{4}\right)\right]}\end{array}$ & $8.99(\mathrm{~s})$ & - & $\begin{array}{l}8.41\left(\mathrm{~d}, 10 \mathrm{H}, \mathrm{H}_{o}\right) \\
7.76\left(\mathrm{~d}, 11 \mathrm{H}, \mathrm{H}_{m, p}\right)\end{array}$ & $2.71\left(\mathrm{~s}, 12 \mathrm{H}, \mathrm{H}_{\mathrm{me}}\right)$ \\
\hline $\begin{array}{l}p-\mathrm{Cl}-\mathrm{Zn}-\mathrm{t}\left(p-\mathrm{CH}_{3}\right) \mathrm{PP} \\
{\left[\left(\mathrm{C}_{6} \mathrm{H}_{4} \mathrm{ClO}\right) \mathrm{Zn}\left(\mathrm{C}_{48} \mathrm{H}_{36} \mathrm{~N}_{4}\right)\right]}\end{array}$ & $9.2(\mathrm{~s})$ & - & $\begin{array}{l}8.29\left(\mathrm{~d}, 10 \mathrm{H}, \mathrm{H}_{o}\right) \\
7.92\left(\mathrm{~d}, 10 \mathrm{H}, \mathrm{H}_{m}\right)\end{array}$ & $2.9\left(\mathrm{~s}, 12 \mathrm{H}, \mathrm{H}_{\mathrm{me}}\right)$ \\
\hline $\begin{array}{l}p-\mathrm{OCH}_{3} \text { phO-Zn-t }\left(p-\mathrm{CH}_{3}\right) \mathrm{PP} \\
{\left[\left(\mathrm{C}_{7} \mathrm{H}_{7} \mathrm{O}_{2}\right) \mathrm{Zn}\left(\mathrm{C}_{48} \mathrm{H}_{36} \mathrm{~N}_{4}\right)\right]}\end{array}$ & $8.93(\mathrm{~s})$ & - & $\begin{array}{l}8.22\left(\mathrm{~d}, 10 \mathrm{H}, \mathrm{H}_{o}\right) \\
7.66\left(\mathrm{~d}, 10 \mathrm{H}, \mathrm{H}_{m}\right)\end{array}$ & $\begin{array}{l}2.31\left(\mathrm{~s}, 12 \mathrm{H}, \mathrm{H}_{\mathrm{me}}\right) \\
3.36\left(\mathrm{~s}, 3 \mathrm{H}, \mathrm{H}_{\mathrm{ome}}\right)\end{array}$ \\
\hline $\begin{array}{l}p-\mathrm{NH}_{2} \mathrm{phO}-\mathrm{Zn}-\mathrm{t}\left(p-\mathrm{CH}_{3}\right) \mathrm{PP} \\
{\left[\left(\mathrm{C}_{6} \mathrm{H}_{6} \mathrm{NO}\right) \mathrm{Zn}\left(\mathrm{C}_{48} \mathrm{H}_{36} \mathrm{~N}_{4}\right)\right]}\end{array}$ & $8.90(\mathrm{~s})$ & - & $\begin{array}{l}8.26\left(\mathrm{~d}, 10 \mathrm{H}, \mathrm{H}_{o}\right) \\
7.69\left(\mathrm{~m}, 10 \mathrm{H}, \mathrm{H}_{m}\right)\end{array}$ & $\begin{array}{l}2.36\left(\mathrm{~s}, 12 \mathrm{H}, \mathrm{H}_{\mathrm{me}}\right) \\
5.09\left(\mathrm{~s}, 2 \mathrm{H}, \mathrm{H}_{\mathrm{NH}_{2}}\right)\end{array}$ \\
\hline $\begin{array}{l}2,4-\mathrm{Cl}_{2} \mathrm{phO}-\mathrm{Zn}-\mathrm{t}\left(p-\mathrm{CH}_{3}\right) \mathrm{PP} \\
{\left[\left(\mathrm{C}_{6} \mathrm{H}_{3} \mathrm{Cl}_{2} \mathrm{O}\right) \mathrm{Zn}\left(\mathrm{C}_{48} \mathrm{H}_{36} \mathrm{~N}_{4}\right)\right]}\end{array}$ & $9.4(\mathrm{~s})$ & - & $\begin{array}{l}8.33\left(\mathrm{~d}, 10 \mathrm{H}, \mathrm{H}_{o}\right) \\
7.96\left(\mathrm{~d}, 10 \mathrm{H}, \mathrm{H}_{m}\right)\end{array}$ & $3.3\left(\mathrm{~s}, 12 \mathrm{H}, \mathrm{H}_{\mathrm{me}}\right)$ \\
\hline $\begin{array}{l}p-\mathrm{NO}_{2} \mathrm{phO}-\mathrm{Zn}-\mathrm{t}\left(p-\mathrm{CH}_{3}\right) \mathrm{PP} \\
{\left[\left(\mathrm{C}_{6} \mathrm{H}_{4} \mathrm{NO}_{3}\right) \mathrm{Zn}\left(\mathrm{C}_{48} \mathrm{H}_{36} \mathrm{~N}_{4}\right)\right]}\end{array}$ & $9.01(\mathrm{~s})$ & - & $\begin{array}{l}8.39\left(\mathrm{~d}, 10 \mathrm{H}, \mathrm{H}_{o}\right) \\
7.92\left(\mathrm{~d}, 10 \mathrm{H}, \mathrm{H}_{m}\right)\end{array}$ & $2.65\left(\mathrm{~s}, 12 \mathrm{H}, \mathrm{H}_{\mathrm{me}}\right)$ \\
\hline
\end{tabular}

${ }^{\mathrm{a}} \delta$ in ppm; the nature of splitting pattern $(\mathrm{s})$ : $(\mathrm{s}=$ singlet, $\mathrm{d}=$ doublet, $\mathrm{t}=$ triplet, and $\mathrm{m}=$ multiplet); number of proton(s) and their location in the porphyrins, respectively, are given in parenthesis; $o=$ ortho; $p=$ para; $m=$ meta.

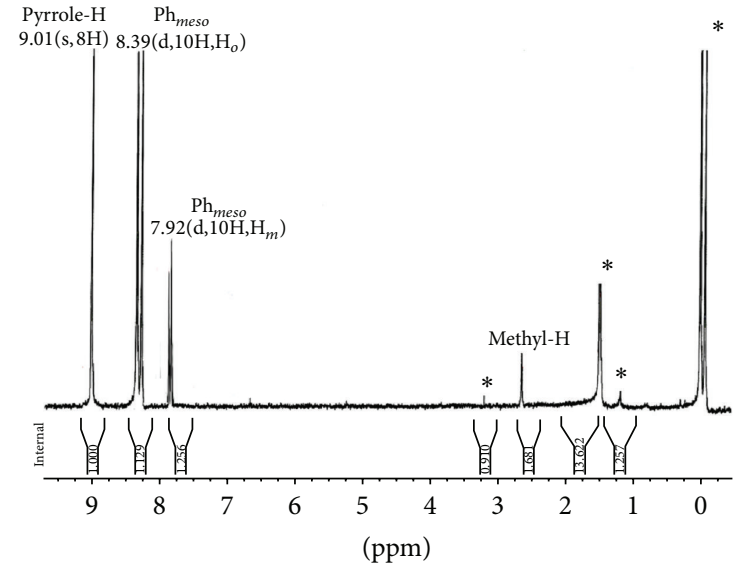

FIgURE 1: ${ }^{1} \mathrm{H}$ NMR spectra of $p-\mathrm{NO}_{2}$ phO-Zn-t- $\left(p-\mathrm{CH}_{3}\right) \mathrm{PP}$ in $\mathrm{CDCl} 3$ at $298 \mathrm{~K}$. Starred peaks are solvents impurities.

The optical absorption spectra of X-Zn-t $\left(p-\mathrm{CH}_{3}\right) \mathrm{PP}(\mathrm{X}=$ different phenols as axial ligand) in chloroform revealed that phenols containing electron withdrawing groups show blue shift (hypsochromic shift) while those having electron releasing groups show red shift (bathochromic shift) that is towards longer wavelength. When absorption spectra of axially ligated $\mathrm{Zn}-\mathrm{t}\left(p-\mathrm{CH}_{3}\right) \mathrm{PP}$ is recorded in different solvents (Table 3), it was observed that the spectra of $p-\mathrm{NH}_{2} \mathrm{phO}$ $\mathrm{Zn}-\mathrm{t}\left(p-\mathrm{CH}_{3}\right) \mathrm{PP}$ (Figure 3 ) shows only a marginal change in $\lambda_{\max }$, absorption coefficient $(\varepsilon)$, and oscillator strength $(f)$ values. The data also reveal that a change in polarity of the solvents does not significantly alter the position of the transition but there is a significant increase in "Fwhm" $\left(\nu_{1 / 2}\right)$ and " $f$ " values of transition by increasing the polarity of the solvent. In polar solvents such as methanol, ethanol, $\mathrm{CH}_{2} \mathrm{Cl}_{2}$,

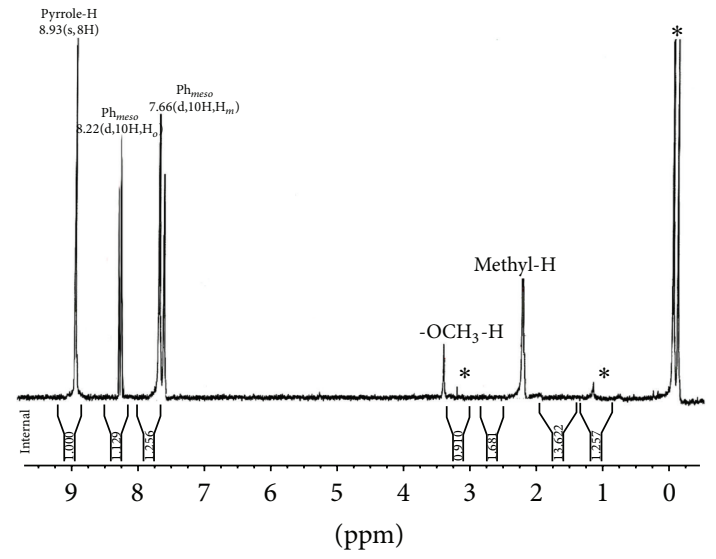

Figure 2: ${ }^{1} \mathrm{H}$ NMR spectra of $p$ - $\mathrm{OCH}_{3}$ phO-Zn-t- $\left(p-\mathrm{CH}_{3}\right) \mathrm{PP}$ in $\mathrm{CDCl}_{3}$ at $298 \mathrm{~K}$. Starred peaks are solvents impurities.

$\mathrm{CHCl}_{3}$, the $\pi \rightarrow \pi^{*}$ band undergoes red shift and was stable but in nonpolar solvents such as benzene, toluene, and $\mathrm{CCl}_{4}$; however, the complexes usually displayed a spectral drift for a period of time. It is observed that, for all the axially ligated $\mathrm{Zn}(\mathrm{II})$ derivatives, B- and Q-bands exhibit a red shift on increasing the polarity of the solvents in the order $\mathrm{MtOH}>\mathrm{CHCl}_{3}>\mathrm{CH}_{2} \mathrm{Cl}_{2}>\mathrm{CCl}_{4}$. As in the case of $p-\mathrm{NH}_{2}$ phO-Zn-t $\left(p-\mathrm{CH}_{3}\right) \mathrm{PP}$ (Figure 3 ), $\lambda_{\max }$ values in $\mathrm{MtOH}$ were observed at $435.9 \mathrm{~nm}, 589 \mathrm{~nm}$, and $604 \mathrm{~nm}$ while in $\mathrm{CHCl}_{3}, \mathrm{CH}_{2} \mathrm{Cl}_{2}$, and $\mathrm{CCl}_{4}$ were observed at $433.9 \mathrm{~nm}$, $570.9 \mathrm{~nm}$, and $604.6 \mathrm{~nm} ; 432.8 \mathrm{~nm}, 568.4 \mathrm{~nm}$, and $601 \mathrm{~nm}$; and $430 \mathrm{~nm}, 560.1 \mathrm{~nm}$, and $586 \mathrm{~nm}$, respectively. However, both $\mathrm{B}(0,0)$ and $\mathrm{Q}(1,0)$ exhibit only a small change in the $f$ value, which depend on the nature of the solvent. The $f$ value for $\mathrm{Q}(1,0)$ in $\mathrm{MtOH}, \mathrm{CHCl}_{3}, \mathrm{CH}_{2} \mathrm{Cl}_{2}$, and $\mathrm{CCl}_{4}$ was observed 
TABLE 2: Optical absorption data of $\mathrm{X}-\mathrm{Zn}-\mathrm{t}\left(p-\mathrm{CH}_{3}\right) \mathrm{PP}(\mathrm{X}=$ different phenols as axial ligand $)$ in $\mathrm{CHCl}_{3}$ showing $\lambda_{\max }$ together with log $\varepsilon$ and $\nu_{1 / 2}$.

\begin{tabular}{|c|c|c|}
\hline Porphyrins & $\begin{array}{c}\text { B-bands } \lambda_{\max },(\log \varepsilon), v_{1 / 2} \\
(\mathrm{~nm}),\left(\mathrm{dm}^{3} \mathrm{~mol}^{-1} \mathrm{~cm}^{-1}\right),\left(\mathrm{cm}^{-1}\right)\end{array}$ & $\begin{array}{c}\text { Q-bands } \lambda_{\max },(\log \varepsilon), v_{1 / 2} \\
(\mathrm{~nm}),\left(\mathrm{dm}^{3} \mathrm{~mol}^{-1} \mathrm{~cm}^{-1}\right),\left(\mathrm{cm}^{-1}\right)\end{array}$ \\
\hline $\begin{array}{l}\mathrm{H}_{2} \mathrm{t}\left(p-\mathrm{CH}_{3}\right) \mathrm{PP} \\
{\left[\left(\mathrm{C}_{48} \mathrm{H}_{38} \mathrm{~N}_{4}\right)\right]}\end{array}$ & $430,(5.986), 989.4$ & $516,553,592,649$ \\
\hline $\begin{array}{l}\mathrm{Zn}-\mathrm{t}\left(p-\mathrm{CH}_{3}\right) \mathrm{PP} \\
{\left[\left(\mathrm{C}_{48} \mathrm{H}_{36} \mathrm{~N}_{4} \mathrm{Zn}_{1}\right)\right]}\end{array}$ & $432,(5.824),(995.3)$ & $\begin{array}{l}564.2,(4.218), 789.3 ; \\
\quad 609.3,(4.160)\end{array}$ \\
\hline $\begin{array}{l}\text { phO-Zn-t }\left(p-\mathrm{CH}_{3}\right) \mathrm{PP} \\
{\left[\left(\mathrm{C}_{6} \mathrm{H}_{5} \mathrm{O}\right) \mathrm{Zn}\left(\mathrm{C}_{48} \mathrm{H}_{36} \mathrm{~N}_{4}\right)\right]}\end{array}$ & $430.2,(5.771),(997.9)$ & $\begin{array}{l}563.8,(4.312), 785.1 \\
\quad 598.6,(4.289)\end{array}$ \\
\hline $\begin{array}{l}\alpha \text {-Naphthol-Zn-t }\left(p-\mathrm{CH}_{3}\right) \mathrm{PP} \\
{\left[\left(\mathrm{C}_{10} \mathrm{H}_{7} \mathrm{O}\right) \mathrm{Zn}\left(\mathrm{C}_{48} \mathrm{H}_{36} \mathrm{~N}_{4}\right)\right]}\end{array}$ & 431.4, (5.798), (1008) & $\begin{array}{l}\text { 564.0, (4.418), 829.4; } \\
\quad 600.4,(4.389)\end{array}$ \\
\hline $\begin{array}{l}o-\mathrm{OCH}_{3} \mathrm{phO}-\mathrm{Zn}-\mathrm{t}\left(p-\mathrm{CH}_{3}\right) \mathrm{PP} \\
{\left[\left(\mathrm{C}_{7} \mathrm{H}_{7} \mathrm{O}_{2}\right) \mathrm{Zn}\left(\mathrm{C}_{48} \mathrm{H}_{36} \mathrm{~N}_{4}\right)\right]}\end{array}$ & $432,(5.833),(980.8)$ & $\begin{array}{l}569.4,(4.484), 826.2 \\
\quad 609.6,(4.338)\end{array}$ \\
\hline $\begin{array}{l}m-\mathrm{OCH}_{3} \text { ph- } \mathrm{Zn}-\mathrm{t}\left(p-\mathrm{CH}_{3}\right) \mathrm{PP} \\
{\left[\left(\mathrm{C}_{7} \mathrm{H}_{7} \mathrm{O}_{2}\right) \mathrm{Zn}\left(\mathrm{C}_{48} \mathrm{H}_{36} \mathrm{~N}_{4}\right)\right]}\end{array}$ & $431.9,(5.845),(978.9)$ & $\begin{array}{l}570.2,(4.521), 789.3 \\
\quad 608.3,(4.432)\end{array}$ \\
\hline $\begin{array}{l}p-\mathrm{OCH}_{3} \mathrm{phO}-\mathrm{Zn}-\mathrm{t}\left(p-\mathrm{CH}_{3}\right) \mathrm{PP} \\
{\left[\left(\mathrm{C}_{7} \mathrm{H}_{7} \mathrm{O}_{2}\right) \mathrm{Zn}\left(\mathrm{C}_{48} \mathrm{H}_{36} \mathrm{~N}_{4}\right)\right]}\end{array}$ & $432.4,(5.808),(984.1)$ & $\begin{array}{l}567,(4.448), 822.4 \\
\quad 604,(4.392)\end{array}$ \\
\hline $\begin{array}{l}o-\mathrm{NH}_{2} \mathrm{phO}-\mathrm{Zn}-\mathrm{t}\left(p-\mathrm{CH}_{3}\right) \mathrm{PP} \\
{\left[\left(\mathrm{C}_{6} \mathrm{H}_{6} \mathrm{NO}\right) \mathrm{Zn}\left(\mathrm{C}_{48} \mathrm{H}_{36} \mathrm{~N}_{4}\right)\right]}\end{array}$ & $433.4,(5.806),(998)$ & $\begin{array}{l}572.4,(4.527), 796.2 \\
\quad 606.3,(4.486)\end{array}$ \\
\hline $\begin{array}{l}m-\mathrm{NH}_{2} \mathrm{phO}-\mathrm{Zn}-\mathrm{t}\left(p-\mathrm{CH}_{3}\right) \mathrm{PP} \\
{\left[\left(\mathrm{C}_{6} \mathrm{H}_{6} \mathrm{NO}\right) \mathrm{Zn}\left(\mathrm{C}_{48} \mathrm{H}_{36} \mathrm{~N}_{4}\right)\right]}\end{array}$ & $433.2,(5.696),(992.4)$ & $\begin{array}{l}570.4,(4.456), 698.6 \\
\quad 605.1,(4.412)\end{array}$ \\
\hline $\begin{array}{l}p-\mathrm{NH}_{2} \mathrm{phO}-\mathrm{Zn}-\mathrm{t}\left(p-\mathrm{CH}_{3}\right) \mathrm{PP} \\
{\left[\left(\mathrm{C}_{6} \mathrm{H}_{6} \mathrm{NO}\right) \mathrm{Zn}\left(\mathrm{C}_{48} \mathrm{H}_{36} \mathrm{~N}_{4}\right)\right]}\end{array}$ & $433.9,(5.859),(986.6)$ & $\begin{array}{l}570.9,(4.432), 699.4 \\
\quad 604.6,(4.398)\end{array}$ \\
\hline $\begin{array}{l}o-\mathrm{CH}_{3} \mathrm{phO}-\mathrm{Zn}-\mathrm{t}\left(p-\mathrm{CH}_{3}\right) \mathrm{PP} \\
{\left[\left(\mathrm{C}_{7} \mathrm{H}_{7} \mathrm{O}\right) \mathrm{Zn}\left(\mathrm{C}_{48} \mathrm{H}_{36} \mathrm{~N}_{4}\right)\right]}\end{array}$ & 431, (5.964), (987.1) & $\begin{array}{l}565,(4.643), 762.1 \\
596,(4.431)\end{array}$ \\
\hline $\begin{array}{l}m-\mathrm{CH}_{3} \mathrm{phO}-\mathrm{Zn}-\mathrm{t}\left(p-\mathrm{CH}_{3}\right) \mathrm{PP} \\
{\left[\left(\mathrm{C}_{7} \mathrm{H}_{7} \mathrm{O}\right) \mathrm{Zn}\left(\mathrm{C}_{48} \mathrm{H}_{36} \mathrm{~N}_{4}\right)\right]}\end{array}$ & $431.8,(5.839),(986.4)$ & $\begin{array}{l}564,(4.682), 760.9 \\
588,(4.432)\end{array}$ \\
\hline $\begin{array}{l}p-\mathrm{CH}_{3} \mathrm{phO}-\mathrm{Zn}-\mathrm{t}\left(p-\mathrm{CH}_{3}\right) \mathrm{PP} \\
{\left[\left(\mathrm{C}_{7} \mathrm{H}_{7} \mathrm{O}\right) \mathrm{Zn}\left(\mathrm{C}_{48} \mathrm{H}_{36} \mathrm{~N}_{4}\right)\right]}\end{array}$ & 431.6, (5.969), (986.9) & $\begin{array}{l}564,(4.861), 760.6 \\
587,(4.743)\end{array}$ \\
\hline $\begin{array}{l}o-\mathrm{NO}_{2} \text { phO-Zn-t }\left(p-\mathrm{CH}_{3}\right) \mathrm{PP} \\
{\left[\left(\mathrm{C}_{6} \mathrm{H}_{4} \mathrm{NO}_{3}\right) \mathrm{Zn}\left(\mathrm{C}_{48} \mathrm{H}_{36} \mathrm{~N}_{4}\right)\right]}\end{array}$ & $428,(5.942),(998.9)$ & $\begin{array}{l}546,(4.549), 854.2 \\
581,(4.431)\end{array}$ \\
\hline $\begin{array}{l}m-\mathrm{NO}_{2} \mathrm{phO}-\mathrm{Zn}-\mathrm{t}\left(p-\mathrm{CH}_{3}\right) \mathrm{PP} \\
{\left[\left(\mathrm{C}_{6} \mathrm{H}_{4} \mathrm{NO}_{3}\right) \mathrm{Zn}\left(\mathrm{C}_{48} \mathrm{H}_{36} \mathrm{~N}_{4}\right)\right]}\end{array}$ & 427, (5.841), (995.2) & $\begin{array}{l}546,(4.643), 846.9 ; \\
580,(4.428)\end{array}$ \\
\hline $\begin{array}{l}p-\mathrm{NO}_{2} \text { phO-Zn-t }\left(p-\mathrm{CH}_{3}\right) \mathrm{PP} \\
{\left[\left(\mathrm{C}_{6} \mathrm{H}_{4} \mathrm{NO}_{3}\right) \mathrm{Zn}\left(\mathrm{C}_{48} \mathrm{H}_{36} \mathrm{~N}_{4}\right)\right]}\end{array}$ & 427, (5.872), (995.6) & $\begin{array}{l}546,(4.516), 842.8 \\
578,(4.314)\end{array}$ \\
\hline $\begin{array}{l}o-\mathrm{ClphO}-\mathrm{Zn}-\mathrm{t}\left(p-\mathrm{CH}_{3}\right) \mathrm{PP} \\
{\left[\left(\mathrm{C}_{6} \mathrm{H}_{4} \mathrm{ClO}\right) \mathrm{Zn}\left(\mathrm{C}_{48} \mathrm{H}_{36} \mathrm{~N}_{4}\right)\right]}\end{array}$ & 426, (5.646), (1014.4) & $\begin{array}{c}547,(4.569), 872.3 ; \\
575,(4.321)\end{array}$ \\
\hline $\begin{array}{l}m \text {-ClphO-Zn-t }\left(p-\mathrm{CH}_{3}\right) \mathrm{PP} \\
{\left[\left(\mathrm{C}_{6} \mathrm{H}_{4} \mathrm{ClO}\right) \mathrm{Zn}\left(\mathrm{C}_{48} \mathrm{H}_{36} \mathrm{~N}_{4}\right)\right]}\end{array}$ & $425,(5.781),(992.5)$ & $\begin{array}{l}548,(4.439), 781.9 ; \\
579,(4.532)\end{array}$ \\
\hline $\begin{array}{l}p \text {-ClphO-Zn-t }\left(p-\mathrm{CH}_{3}\right) \mathrm{PP} \\
{\left[\left(\mathrm{C}_{6} \mathrm{H}_{4} \mathrm{ClO}\right) \mathrm{Zn}\left(\mathrm{C}_{48} \mathrm{H}_{36} \mathrm{~N}_{4}\right)\right]}\end{array}$ & $425,(5.841),(998.6)$ & $\begin{array}{c}548,(4.598), 785.2 \\
579,(4.514)\end{array}$ \\
\hline
\end{tabular}

at $0.206755,0.253434,0.1878264$, and 0.1331380 , respectively. It was found that, with the increase in polarity of the solvents, the axially ligated $\mathrm{Zn}$ (II) metalloporphyrin with different phenols as axial ligand shows the progressive broadening of the B- and Q-bands indicating that the magnitude of change of the " $f$ " value depends on the nature of the solvent and also reveals the relative strength of $\pi \rightarrow \pi^{*}$ interactions.

3.1.3. Infrared Spectroscopy. The vibrational spectroscopy (IR spectroscopy) can provide ample information about the structure of porphyrin and metalloporphyrin. The IR spectra of free-base porphyrin, $\mathrm{H}_{2}-\mathrm{t}\left(p-\mathrm{CH}_{3}\right) \mathrm{PP}$, and its axially ligated zinc(II) metal derivatives containing different phenols as axial ligand exhibit strong absorption band at $2855 \mathrm{~cm}^{-1}$ $\left(2850-3000 \mathrm{~cm}^{-1}\right)$ due to $-\mathrm{CH}_{3}$ group at meso-phenyl position. The metallation of porphyrin and axial ligation with different phenols were further supported by the emergence of two new bands at $500-400 \mathrm{~cm}^{-1}$ and $650-350 \mathrm{~cm}^{-1}$ assigned to zinc-nitrogen ( $\left.\mathrm{Zn}-\mathrm{N}_{\text {Por }}\right)$ [41] and zinc-oxygen ( $\left.\mathrm{Zn}-\mathrm{O}\right)$ vibrational modes, respectively. In order to determine the mode of bonding of different phenols as ligand with zinc, the IR spectra of ligands were compared with those of corresponding complexes (Table 4). The IR spectra of free-base porphyrin $\left(\mathrm{H}_{2}-\mathrm{t}\left(p-\mathrm{CH}_{3}\right) \mathrm{PP}\right)$ and its axially ligated zinc(II) metal derivatives containing different phenols as axial ligand showing different band frequencies agree well with 
TABLE 3: Optical absorption data of X-Zn-t $\left(p-\mathrm{CH}_{3}\right) \mathrm{PP}(\mathrm{X}=$ different phenols as axial ligand $)$ recorded in different solvents with calculated " $f$ " values.

\begin{tabular}{|c|c|c|c|c|c|c|}
\hline \multirow[t]{2}{*}{ Porphyrins } & \multirow[t]{2}{*}{ Solvent } & \multirow{2}{*}{$\begin{array}{l}\text { B-band } \lambda_{\max },(\log \varepsilon), v_{1 / 2} \\
(\mathrm{~nm}),\left(\mathrm{dm}^{3} \mathrm{~mol}^{-1} \mathrm{~cm}^{-1}\right), \\
\left(\mathrm{cm}^{-1}\right)\end{array}$} & \multicolumn{2}{|c|}{$\begin{array}{l}\text { Q-bands } \lambda_{\max },(\log \varepsilon), v_{1 / 2}(\mathrm{~nm}) \\
\left(\mathrm{dm}^{3} \mathrm{~mol}^{-1} \mathrm{~cm}^{-1}\right),\left(\mathrm{cm}^{-1}\right)\end{array}$} & \multicolumn{2}{|c|}{$\begin{array}{l}\text { Oscillator strength } \\
\left(f=4.33 \times 10^{-9} \varepsilon \Delta v_{1 / 2}\right)\end{array}$} \\
\hline & & & $\mathrm{Q}(1,0)$ & $\mathrm{Q}(0,0)$ & B-band & $\begin{array}{l}\text { Q-band } \\
\text { Q }(1,0)\end{array}$ \\
\hline \multirow{4}{*}{$\begin{array}{l}p-\mathrm{NH}_{2} \mathrm{phO}-\mathrm{Zn}-\mathrm{t}\left(p-\mathrm{CH}_{3}\right) \mathrm{PP} \\
{\left[\left(\mathrm{C}_{6} \mathrm{H}_{6} \mathrm{NO}\right) \mathrm{Zn}\left(\mathrm{C}_{48} \mathrm{H}_{36} \mathrm{~N}_{4}\right)\right]}\end{array}$} & $\mathrm{MtOH}$ & $\begin{array}{c}435.9,714 \\
(5.638), 45489\end{array}$ & $\begin{array}{c}589,925 \\
(4.724), 51621\end{array}$ & $\begin{array}{r}604,531 \\
(4.604)\end{array}$ & 0.140635 & 0.206755 \\
\hline & $\mathrm{CHCl}_{3}$ & $\begin{array}{c}433.9,437 \\
(5.329), 23458\end{array}$ & $\begin{array}{c}570.9,1096 \\
(4.749), 53403\end{array}$ & $\begin{array}{c}604.6,532 \\
(4.598)\end{array}$ & 0.044387 & 0.253434 \\
\hline & $\mathrm{CH}_{2} \mathrm{Cl}_{2}$ & $\begin{array}{c}432.8,542 \\
(5.261), 18609\end{array}$ & $\begin{array}{c}568.4,679 \\
(4.896), 63885\end{array}$ & $\begin{array}{c}601,740 \\
(4.423)\end{array}$ & 0.0436727 & 0.1878264 \\
\hline & $\mathrm{CCl}_{4}$ & $\begin{array}{c}430,631 \\
(5.594), 42352\end{array}$ & $\begin{array}{c}560.1,594 \\
(4.726), 51764\end{array}$ & $\begin{array}{r}586,447 \\
(4.412)\end{array}$ & 0.115715 & 0.1331380 \\
\hline \multirow{4}{*}{$\begin{array}{l}m-\mathrm{ClphO}-\mathrm{Zn}-\mathrm{t}\left(p-\mathrm{CH}_{3}\right) \mathrm{PP} \\
{\left[\left(\mathrm{C}_{6} \mathrm{H}_{4} \mathrm{ClO}\right) \mathrm{Zn}\left(\mathrm{C}_{48} \mathrm{H}_{36} \mathrm{~N}_{4}\right)\right]}\end{array}$} & $\mathrm{MtOH}$ & $\begin{array}{c}433.4,615 \\
(5.964), 71749\end{array}$ & $\begin{array}{c}564,459 \\
(4.643), 47858\end{array}$ & $\begin{array}{c}592,525 \\
(4.428)\end{array}$ & 0.19106399 & 0.0951163 \\
\hline & $\mathrm{CHCl}_{3}$ & $\begin{array}{c}425,750 \\
(5.781), 58129\end{array}$ & $\begin{array}{c}548,455 \\
(4.439), 32674\end{array}$ & $\begin{array}{c}579,549 \\
(4.532)\end{array}$ & 0.1807739 & 0.0643726 \\
\hline & $\mathrm{CH}_{2} \mathrm{Cl}_{2}$ & $\begin{array}{c}424,857 \\
(5.536), 39894\end{array}$ & $\begin{array}{c}546,426 \\
(4.552), 41085\end{array}$ & $\begin{array}{c}573,503 \\
(4.561)\end{array}$ & 0.14803905 & 0.0757845 \\
\hline & $\mathrm{CCl}_{4}$ & $\begin{array}{c}422.8,820 \\
(5.536), 39894 \\
\end{array}$ & $\begin{array}{c}542,561 \\
(4.742), 55227 \\
\end{array}$ & $\begin{array}{c}568,511 \\
(4.431) \\
\end{array}$ & 0.1416476 & 0.1341536 \\
\hline \multirow{4}{*}{$\begin{array}{l}m-\mathrm{NO}_{2} \mathrm{phOZn}-\mathrm{t}\left(p-\mathrm{CH}_{3}\right) \mathrm{PP} \\
{\left[\left(\mathrm{C}_{6} \mathrm{H}_{4} \mathrm{NO}_{3}\right) \mathrm{Zn}\left(\mathrm{C}_{48} \mathrm{H}_{36} \mathrm{~N}_{4}\right)\right]}\end{array}$} & $\mathrm{MtOH}$ & $\begin{array}{c}429,686 \\
(5.389), 29681\end{array}$ & $\begin{array}{c}584,485 \\
(4.643), 49061\end{array}$ & $\begin{array}{c}616.2,555 \\
(4.222)\end{array}$ & 0.0881638 & 0.1030306 \\
\hline & $\mathrm{CHCl}_{3}$ & $\begin{array}{c}427,770 \\
(5.872), 66534\end{array}$ & $\begin{array}{c}546,426 \\
(4.643), 49061\end{array}$ & $\begin{array}{c}580,463 \\
(4.428)\end{array}$ & 0.2218310 & 0.0904969 \\
\hline & $\mathrm{CH}_{2} \mathrm{Cl}_{2}$ & $\begin{array}{c}427.8,607 \\
(5.418), 31893\end{array}$ & $\begin{array}{c}544.8,470 \\
(4.549), 41889\end{array}$ & $\begin{array}{c}577.4,367 \\
(4.431)\end{array}$ & 0.0838246 & 0.0852483 \\
\hline & $\mathrm{CCl}_{4}$ & $\begin{array}{c}424.4,766 \\
(5.569), 43415\end{array}$ & $\begin{array}{c}542.9,540 \\
(4.321), 24493\end{array}$ & $\begin{array}{c}540.2,584 \\
(4.249)\end{array}$ & 0.1439980 & 0.0572695 \\
\hline
\end{tabular}

the literature [42]. For example, in the IR spectra of $2,4-\mathrm{Cl}_{2}$ phO- $\mathrm{Zn}-\mathrm{t}\left(p-\mathrm{CH}_{3}\right) \mathrm{PP}$, aromatic $\nu(\mathrm{C}-\mathrm{H})$ vibrates at $2963 \mathrm{~cm}^{-1}, \nu(\mathrm{C}-\mathrm{N})$ at $1349 \mathrm{~cm}^{-1}, \nu(\mathrm{C}=\mathrm{C})$ at $1659 \mathrm{~cm}^{-1}$, $\nu(\mathrm{C}=\mathrm{N})$ at $1594 \mathrm{~cm}^{-1}, v\left(\mathrm{CH}_{3}\right)$ at $2846 \mathrm{~cm}^{-1}$, and $\nu\left(\mathrm{Zn}-\mathrm{N}_{\text {Por }}\right)$ at $476 \mathrm{~cm}^{-1}$ and $\nu(\mathrm{Zn}-\mathrm{O})$ of phenolate appears at $517 \mathrm{~cm}^{-1}$ and in $p-\mathrm{OCH}_{3}$ phO- $\mathrm{Zn}-\mathrm{t}\left(p-\mathrm{CH}_{3}\right) \mathrm{PP}$, aromatic $v(\mathrm{C}-\mathrm{H})$ vibrates at $2964 \mathrm{~cm}^{-1}, \nu(\mathrm{C}-\mathrm{N})$ at $1350 \mathrm{~cm}^{-1}, \nu(\mathrm{C}=\mathrm{C})$ at $1654 \mathrm{~cm}^{-1}$, $\nu(\mathrm{C}=\mathrm{N})$ at $1590 \mathrm{~cm}^{-1}, \nu\left(\mathrm{CH}_{3}\right)$ at $2859 \mathrm{~cm}^{-1}, \nu\left(\mathrm{Zn}-\mathrm{N}_{\text {Por }}\right)$ at $481 \mathrm{~cm}^{-1}, v(\mathrm{Zn}-\mathrm{O})$ of phenolate at $526 \mathrm{~cm}^{-1}$ and the methoxy $\left(-\mathrm{OCH}_{3}\right)$ group at the para-position of the phenolate vibrates at $2850 \mathrm{~cm}^{-1}$ for $\left(\nu_{1}\right)(\mathrm{C}-\mathrm{H}), 1020 \mathrm{~cm}^{-1}$ for $\left(\nu_{2}\right)(\mathrm{C}-\mathrm{O}-\mathrm{C})_{\mathrm{sym}}$, and $1261 \mathrm{~cm}^{-1}$ for $\left(\nu_{3}\right)(\mathrm{C}-\mathrm{O}-\mathrm{C})_{\mathrm{asym}}$, respectively. The formations of the axially ligated $\mathrm{Zn}$ (II), metal complexes were also confirmed by their mass spectral data given in the experimental section.

3.1.4. Mass Spectroscopy and Elemental Analysis. The mass spectra of several porphyrins and their metalloderivatives have been obtained by mass spectroscopy technique. The molecular mass [43] spectra of porphyrins and their derivatives are best recorded at the lowest possible temperature (usually approx. $200-250^{\circ} \mathrm{C}$ ). The intensity of molecular ion in the mass spectra of porphyrin has also been used in the study of deuteration (as a general example of electrophilic substitution) of porphyrins, metalloporphyrins, and some reduced derivatives. Table 5 summarizes the mass spectra and elemental analysis data of axially ligated $\mathrm{Zn}$ (II) derivates.

3.1.5. Fluorescence Spectroscopy. An important and unique feature of porphyrins and their metal derivatives is their emission spectra. The fluorescence emission data $[44,45]$ of the porphyrins provide important information on the singlet excited state properties. The optical properties are affected by the presence of substituents at $\beta$-pyrrole and at meso-aryl positions of the tetraphenylporphyrins. The axially ligated metalloporphyrins exhibited two fluorescence bands, one from $S_{2} \rightarrow S_{0}$ (B-band) and the other from $S_{1} \rightarrow$ $S_{0}$ (Q-band). Internal conversion from $S_{2}$ to $S_{1}$ is rapid so that there is hardly any fluorescence absorption detected from $\mathrm{S}_{2} \rightarrow \mathrm{S}_{1}$. The $\mathrm{S}_{2} \rightarrow \mathrm{S}_{0}$ (soret band) fluorescence is about two orders of magnitude weaker than $\mathrm{S}_{1} \rightarrow \mathrm{S}_{0}$ of Q-band emission. However, the emission bands of axially ligated $\mathrm{Zn}$ (II) porphyrins are red shifted compared to $\mathrm{Zn}-\mathrm{t}\left(p-\mathrm{CH}_{3}\right) \mathrm{PP}$. The intensities of low energy $\mathrm{Q}(1,0)$ are more intense than high energy $\mathrm{Q}(0,0)$ band in contrast to that observed for the free-base porphyrins. On comparing the fluorescence behavior of $\mathrm{Zn}-\mathrm{t}\left(p-\mathrm{CH}_{3}\right) \mathrm{PP}$ with $p-\mathrm{NH}_{2}$ phO- $\mathrm{Zn}-\mathrm{t}\left(p-\mathrm{CH}_{3}\right) \mathrm{PP}$ in dry methanol at room temperature using excitation at $\sim 550 \mathrm{~nm}$ (Table 6) (Figure 4), it is clear from the figure that 
TABLE 4: Main vibrational frequencies corresponding to the various groups in $\mathrm{X}-\mathrm{Zn}-\mathrm{t}\left(p-\mathrm{CH}_{3}\right) \mathrm{PP}(\mathrm{X}=$ different phenols as axial ligand).

\begin{tabular}{|c|c|c|c|c|c|c|c|c|c|}
\hline Porphyrins & $\begin{array}{c}v(\mathrm{~N}-\mathrm{H}) \\
\left(\mathrm{cm}^{-1}\right)\end{array}$ & $\begin{array}{c}v(\mathrm{C}-\mathrm{H}) \\
\left(\mathrm{cm}^{-1}\right)\end{array}$ & $\begin{array}{c}v(\mathrm{C}-\mathrm{N}) \\
\left(\mathrm{cm}^{-1}\right)\end{array}$ & $\begin{array}{l}v(\mathrm{C}=\mathrm{C}) \\
\left(\mathrm{cm}^{-1}\right)\end{array}$ & $\begin{array}{c}v(\mathrm{C}=\mathrm{N}) \\
\left(\mathrm{cm}^{-1}\right)\end{array}$ & $\begin{array}{l}v\left(\mathrm{CH}_{3}\right) \\
\left(\mathrm{cm}^{-1}\right)\end{array}$ & $\begin{array}{c}v\left(\mathrm{Zn}-\mathrm{N}_{\text {por }}\right) \\
\left(\mathrm{cm}^{-1}\right)\end{array}$ & $\begin{array}{c}v(\mathrm{Zn}-\mathrm{O}) \\
\left(\mathrm{cm}^{-1}\right)\end{array}$ & Other assignments \\
\hline $\begin{array}{l}\mathrm{H}_{2} \mathrm{t}\left(p-\mathrm{CH}_{3}\right) \mathrm{PP} \\
{\left[\left(\mathrm{C}_{48} \mathrm{H}_{38} \mathrm{~N}_{4}\right)\right]} \\
\end{array}$ & 3446 & 2964 & 1350 & 1650 & 1589 & 2855 & - & - & \\
\hline $\begin{array}{l}\mathrm{Zn}-\mathrm{t}\left(p-\mathrm{CH}_{3}\right) \mathrm{PP} \\
{\left[\left(\mathrm{C}_{48} \mathrm{H}_{36} \mathrm{~N}_{4} \mathrm{Zn}_{1}\right)\right]}\end{array}$ & - & 2963 & 1349 & 1658 & 1594 & 2849 & 482 & - & \\
\hline $\begin{array}{l}\text { phO-Zn-t }\left(p-\mathrm{CH}_{3}\right) \mathrm{PP} \\
{\left[\left(\mathrm{C}_{6} \mathrm{H}_{5} \mathrm{O}\right) \mathrm{Zn}\left(\mathrm{C}_{48} \mathrm{H}_{36} \mathrm{~N}_{4}\right)\right]}\end{array}$ & - & 2963 & 1351 & 1654 & 1590 & 2851 & 473 & 519 & \\
\hline $\begin{array}{l}p-\mathrm{OCH}_{3} \text { phO-Zn-t }(p- \\
\left.\mathrm{CH}_{3}\right) \mathrm{PP} \\
{\left[\left(\mathrm{C}_{7} \mathrm{H}_{7} \mathrm{O}_{2}\right) \mathrm{Zn}\left(\mathrm{C}_{48} \mathrm{H}_{36} \mathrm{~N}_{4}\right)\right]}\end{array}$ & - & 2964 & 1350 & 1649 & 1586 & 2859 & 481 & 526 & $\begin{array}{c}v_{1}(\mathrm{C}-\mathrm{H})=2850 \\
v_{2}(\mathrm{C}-\mathrm{O}-\mathrm{C})_{\text {sym }}=1020 \\
v_{3}(\mathrm{C}-\mathrm{O}-\mathrm{C})_{\text {asym }}=1261\end{array}$ \\
\hline $\begin{array}{l}p-\mathrm{NH}_{2} \text { phO-Zn-t }\left(p-\mathrm{CH}_{3}\right) \mathrm{PP} \\
{\left[\left(\mathrm{C}_{6} \mathrm{H}_{6} \mathrm{NO}\right) \mathrm{Zn}\left(\mathrm{C}_{48} \mathrm{H}_{36} \mathrm{~N}_{4}\right)\right]} \\
\end{array}$ & - & 2963 & 1351 & 1646 & 1583 & 2856 & 464 & 521 & $\begin{array}{l}v_{1}\left(\mathrm{NH}_{2}\right)_{\text {sym }}=3290 \\
v_{2}\left(\mathrm{NH}_{2}\right)_{\mathrm{asym}}=3356\end{array}$ \\
\hline $\begin{array}{l}p-\mathrm{NO}_{2} \text { phO-Zn-t }\left(p-\mathrm{CH}_{3}\right) \mathrm{PP} \\
{\left[\left(\mathrm{C}_{6} \mathrm{H}_{4} \mathrm{NO}_{3}\right) \mathrm{Zn}\left(\mathrm{C}_{48} \mathrm{H}_{36} \mathrm{~N}_{4}\right)\right]}\end{array}$ & - & 2963 & 1350 & 1658 & 1592 & 2851 & 483 & 518 & $\begin{array}{l}v_{1}\left(\mathrm{NO}_{2}\right)_{\text {sym }}=1344 \\
v_{2}\left(\mathrm{NO}_{2}\right)_{\text {asym }}=1527\end{array}$ \\
\hline $\begin{array}{l}2,4-\mathrm{Cl}_{2} \mathrm{phO}-\mathrm{Zn}-\mathrm{t}\left(p-\mathrm{CH}_{3}\right) \mathrm{PP} \\
{\left[\left(\mathrm{C}_{6} \mathrm{H}_{3} \mathrm{Cl}_{2} \mathrm{O}\right) \mathrm{Zn}\left(\mathrm{C}_{48} \mathrm{H}_{36} \mathrm{~N}_{4}\right)\right]}\end{array}$ & - & 2963 & 1349 & 1659 & 1594 & 2846 & 476 & 517 & \\
\hline $\begin{array}{l}p-\mathrm{CH}_{3}-\mathrm{phO}-\mathrm{Zn}-\mathrm{t}\left(p-\mathrm{CH}_{3}\right) \mathrm{PP} \\
{\left[\left(\mathrm{C}_{7} \mathrm{H}_{7} \mathrm{O}\right) \mathrm{Zn}\left(\mathrm{C}_{48} \mathrm{H}_{36} \mathrm{~N}_{4}\right)\right]}\end{array}$ & - & 2951 & 1346 & 1642 & 1580 & 2862 & 468 & 519 & \\
\hline
\end{tabular}

TABle 5: Mass data $(m / z$ ratio $)$ and elemental analytical data of X-Zn-t $\left(p-\mathrm{CH}_{3}\right) \mathrm{PP}(\mathrm{X}=$ different phenols as axial ligand) along with their calculated values.

\begin{tabular}{|c|c|c|c|c|c|}
\hline \multirow{2}{*}{ Porphyrins } & \multirow{2}{*}{ Molecular formula } & \multirow{2}{*}{$\begin{array}{l}m / z \text { ratio } \\
\text { calculated (found) }\end{array}$} & \multicolumn{3}{|c|}{ Percentage calculated (found) } \\
\hline & & & $\mathrm{C}$ & $\mathrm{H}$ & $\mathrm{N}$ \\
\hline $\begin{array}{l}\text { phO-Zn-t }\left(p-\mathrm{CH}_{3}\right) \mathrm{PP} \\
{\left[\left(\mathrm{C}_{6} \mathrm{H}_{5} \mathrm{O}\right) \mathrm{Zn}\left(\mathrm{C}_{48} \mathrm{H}_{36} \mathrm{~N}_{4}\right)\right]}\end{array}$ & $\mathrm{C}_{54} \mathrm{H}_{41} \mathrm{~N}_{4} \mathrm{ZnO}$ & $\begin{array}{c}828.39 \\
(828.29)\end{array}$ & $\begin{array}{c}78.29 \\
(78.30)\end{array}$ & $\begin{array}{l}4.99 \\
(4.99)\end{array}$ & $\begin{array}{l}6.76 \\
(6.76)\end{array}$ \\
\hline $\begin{array}{l}\alpha \text {-Naphthol-Zn-t }\left(p-\mathrm{CH}_{3}\right) \mathrm{PP} \\
{\left[\left(\mathrm{C}_{10} \mathrm{H}_{7} \mathrm{O}\right) \mathrm{Zn}\left(\mathrm{C}_{48} \mathrm{H}_{36} \mathrm{~N}_{4}\right)\right]}\end{array}$ & $\mathrm{C}_{58} \mathrm{H}_{43} \mathrm{~N}_{4} \mathrm{ZnO}$ & $\begin{array}{c}878.90 \\
(878.96)\end{array}$ & $\begin{array}{c}79.31 \\
(79.17)\end{array}$ & $\begin{array}{l}4.94 \\
(4.93)\end{array}$ & $\begin{array}{l}6.38 \\
(6.37)\end{array}$ \\
\hline $\begin{array}{l}p-\mathrm{NH}_{2} \mathrm{phO}-\mathrm{Zn}-\mathrm{t}\left(p-\mathrm{CH}_{3}\right) \mathrm{PP} \\
{\left[\left(\mathrm{C}_{6} \mathrm{H}_{6} \mathrm{NO}\right) \mathrm{Zn}\left(\mathrm{C}_{48} \mathrm{H}_{36} \mathrm{~N}_{4}\right)\right]}\end{array}$ & $\mathrm{C}_{54} \mathrm{H}_{43} \mathrm{~N}_{5} \mathrm{ZnO}$ & $\begin{array}{c}843.39 \\
(844.46)\end{array}$ & $\begin{array}{c}76.81 \\
(76.85)\end{array}$ & $\begin{array}{l}5.13 \\
(5.14)\end{array}$ & $\begin{array}{c}8.3 \\
(8.30)\end{array}$ \\
\hline $\begin{array}{l}o-\mathrm{OCH}_{3} \text { phO-Zn-t }\left(p-\mathrm{CH}_{3}\right) \mathrm{PP} \\
{\left[\left(\mathrm{C}_{7} \mathrm{H}_{7} \mathrm{O}_{2}\right) \mathrm{Zn}\left(\mathrm{C}_{48} \mathrm{H}_{36} \mathrm{~N}_{4}\right)\right]}\end{array}$ & $\mathrm{C}_{55} \mathrm{H}_{43} \mathrm{~N}_{4} \mathrm{ZnO}_{2}$ & $\begin{array}{c}858.53 \\
(858.49)\end{array}$ & $\begin{array}{c}76.94 \\
(76.77)\end{array}$ & $\begin{array}{l}5.05 \\
(5.03)\end{array}$ & $\begin{array}{l}6.53 \\
(6.51)\end{array}$ \\
\hline $\begin{array}{l}m \text {-ClphO-Zn-t }\left(p-\mathrm{CH}_{3}\right) \mathrm{PP} \\
{\left[\left(\mathrm{C}_{6} \mathrm{H}_{4} \mathrm{ClO}\right) \mathrm{Zn}\left(\mathrm{C}_{48} \mathrm{H}_{36} \mathrm{~N}_{4}\right)\right]}\end{array}$ & $\mathrm{C}_{54} \mathrm{H}_{40} \mathrm{~N}_{4} \mathrm{ZnOCl}$ & $\begin{array}{c}862.95 \\
(862.90)\end{array}$ & $\begin{array}{c}75.16 \\
(75.08)\end{array}$ & $\begin{array}{l}4.67 \\
(4.67)\end{array}$ & $\begin{array}{c}6.49 \\
(6.49)\end{array}$ \\
\hline $\begin{array}{l}2,4 \mathrm{Cl}_{2} \mathrm{phO}-\mathrm{Zn}-\mathrm{t}\left(p-\mathrm{CH}_{3}\right) \mathrm{PP} \\
{\left[\left(\mathrm{C}_{6} \mathrm{H}_{3} \mathrm{Cl}_{2} \mathrm{O}\right) \mathrm{Zn}\left(\mathrm{C}_{48} \mathrm{H}_{36} \mathrm{~N}_{4}\right)\right]}\end{array}$ & $\mathrm{C}_{54} \mathrm{H}_{39} \mathrm{~N}_{4} \mathrm{ZnOCl}_{2}$ & $\begin{array}{l}897.39 \\
(897.30)\end{array}$ & $\begin{array}{c}72.27 \\
(72.36)\end{array}$ & $\begin{array}{c}4.38 \\
(4.39)\end{array}$ & $\begin{array}{c}6.24 \\
(6.25) \\
\end{array}$ \\
\hline
\end{tabular}

the emission bands of $p-\mathrm{NH}_{2}$ phO- $\mathrm{Zn}-\mathrm{t}\left(p-\mathrm{CH}_{3}\right) \mathrm{PP}$ are red shifted compared to $\mathrm{Zn}-\mathrm{t}\left(p-\mathrm{CH}_{3}\right) \mathrm{PP}$ which is due to the electron donating effect of the amino $\left(-\mathrm{NH}_{2}\right)$ group attached to the phenolate ion. This fluorescence analysis procedure is of great importance in identifying the porphyrin chemosensors for selective detection of amine compounds of biological and technical interest. In addition, the possibilities of producing solid-state solar cells by synthesis of semiconductors with porphyrin compounds have been intensely explored.

\subsubsection{TGA/DTG Studies}

Thermal Analysis of $p-\mathrm{OCH}_{3} \mathrm{phO}-\mathrm{Zn-t}-\left(\mathrm{p}-\mathrm{CH}_{3}\right) \mathrm{PP}$. Thermogravimetric analyses were performed in an air atmosphere 
TABLE 6: Fluorescence spectral data of axially ligated compound of $\mathrm{X}-\mathrm{Zn}-\mathrm{t}\left(p-\mathrm{CH}_{3}\right) \mathrm{PP}$ ( $\mathrm{X}=$ different phenols as axial ligands) in methanol solvent using excitation at $\sim 550 \mathrm{~nm}$.

\begin{tabular}{lc}
\hline Porphyrins & $\lambda_{\max }(\mathrm{nm})$ \\
\hline $\mathrm{Zn}-\mathrm{t}\left(p-\mathrm{CH}_{3}\right) \mathrm{PP}$ & 590,640 \\
{$\left[\left(\mathrm{C}_{48} \mathrm{H}_{36} \mathrm{~N}_{4} \mathrm{Zn} \mathrm{n}_{1}\right)\right]$} & \\
$p-\mathrm{NH}_{2} \mathrm{phO}-\mathrm{Zn}-\mathrm{t}\left(p-\mathrm{CH}_{3}\right) \mathrm{PP}$ & 612,660 \\
{$\left[\left(\mathrm{C}_{6} \mathrm{H}_{6} \mathrm{NO}\right) \mathrm{Zn}\left(\mathrm{C}_{48} \mathrm{H}_{36} \mathrm{~N}_{4}\right)\right]$} & \\
\hline
\end{tabular}

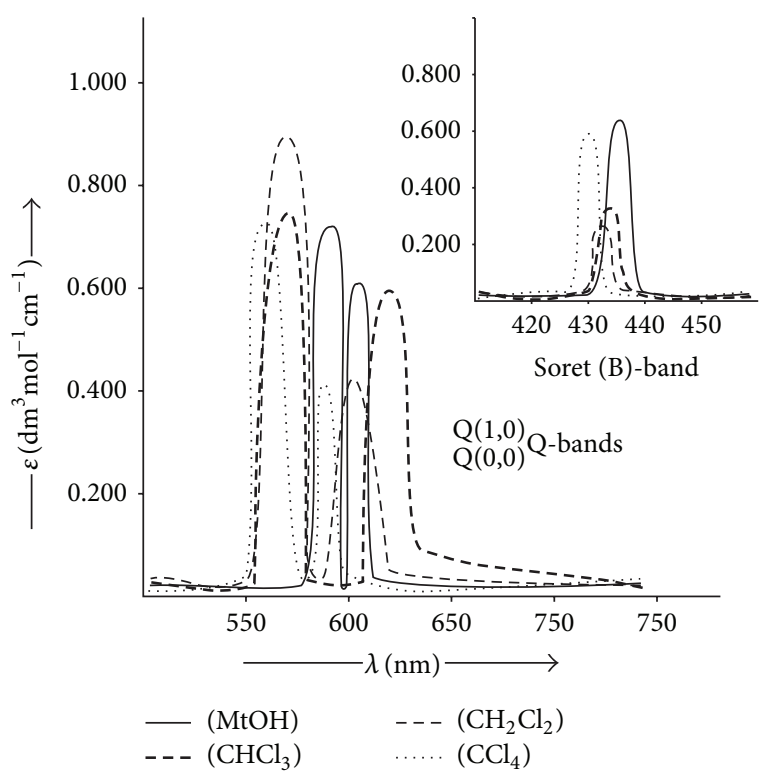

Figure 3: UV-Visible spectra of $p-\mathrm{NH}_{2} \mathrm{phO}-\mathrm{Zn}-\mathrm{t}\left(p-\mathrm{CH}_{3}\right) \mathrm{PP}$ in different solvents.

at a heating rate of $10^{\circ} \mathrm{C} / \mathrm{min}$ to examine thermal stability of the compound. The TG curve of the complex $p-\mathrm{OCH}_{3} \mathrm{phO}-$ $\mathrm{Zn}-\mathrm{t}-\left(p-\mathrm{CH}_{3}\right) \mathrm{PP}$ (Figure 5) shows a continuous weight loss starting from $150^{\circ} \mathrm{C}$ to $800^{\circ} \mathrm{C}$, when a stable oxide of $\mathrm{ZnO}$ is formed. The TG curve shows an initial weight loss of about $14.34 \%$ (the theoretical value $=14.7 \%$ ) observed between $140^{\circ} \mathrm{C}$ and $170^{\circ} \mathrm{C}$ and is attributed to the removal of paramethoxy-phenyl ring as axial group. In the range of $200^{\circ} \mathrm{C}$ to $425^{\circ} \mathrm{C}$, up to $42.2 \%$ of the mass had been lost due to the loss of tetraphenyl group (the theoretical value $=42.39 \%$ ). At $446.0^{\circ} \mathrm{C}$, up to $69.90 \%$ (the theoretical value $=70.79 \%$ ) of the total mass had been lost, corresponding to the collapse of macrocyclic ligand. The organic moiety decomposes further with increasing temperature. Further in the range of $450^{\circ} \mathrm{C}-$ $600^{\circ} \mathrm{C}$, the weight loss reaches up to $96.7 \%$ which is attributed to the removal of pyrrole groups and complete decomposition of macrocyclic ligand and finally $\mathrm{ZnO}$ is remained (the theoretical value $=96.9 \%$ ).

Simultaneously, there were three exothermal peaks at $492^{\circ} \mathrm{C}, 563^{\circ} \mathrm{C}$, and $580^{\circ} \mathrm{C}$ on the DTA curve, corresponding to the major weight loss in the ligand $\mathrm{H}_{2}-\mathrm{t}\left(p-\mathrm{CH}_{3}\right) \mathrm{PP}$ $\left(460^{\circ} \mathrm{C}-580^{\circ} \mathrm{C}\right)$. The small exothermic peak corresponds to the decomposition of the ligand and the loss of chains of

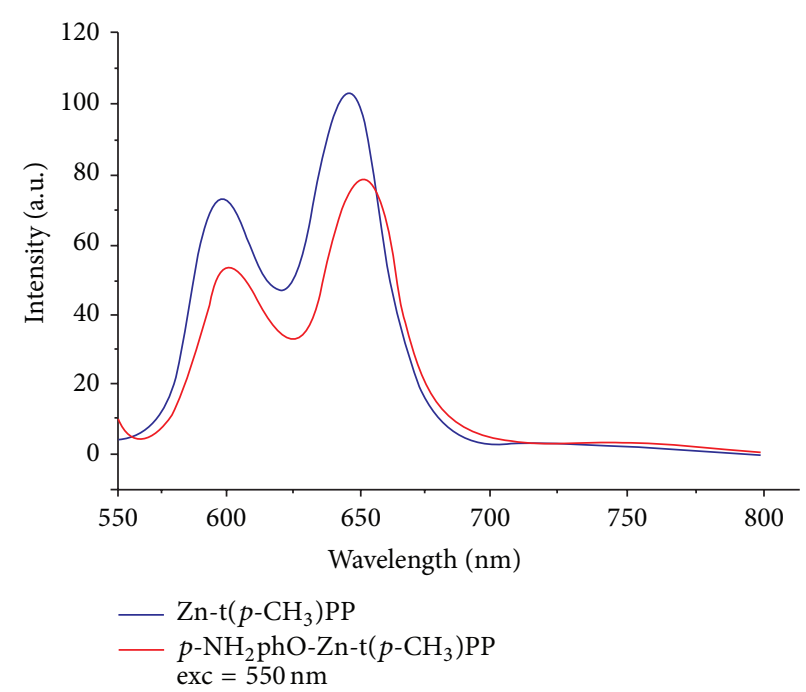

Figure 4: Fluorescence spectral data of $\mathrm{Zn}-\mathrm{t}\left(p-\mathrm{CH}_{3}\right) \mathrm{PP}$ and $p$ $\mathrm{NH}_{2}$ phO-Zn-t $\left(p-\mathrm{CH}_{3}\right) \mathrm{PP}$ in methanol at excitation $550 \mathrm{~nm}$.

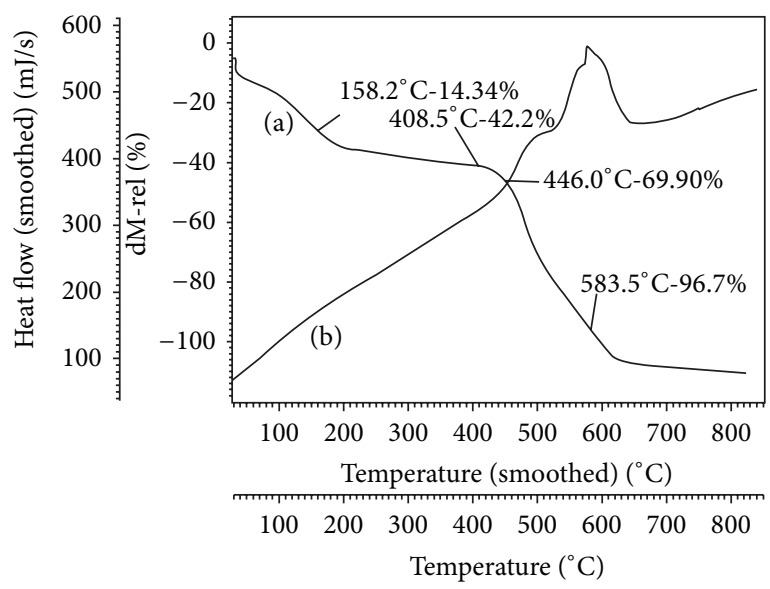

FIGURE 5: TG curve (a) and DTA curve (b) of $p-\mathrm{OCH}_{3}$ phO-Zn-t $(p-$ $\left.\mathrm{CH}_{3}\right) \mathrm{PP}$.

the porphyrin ring and the large exothermic peak corresponds to the collapse of the porphyrin skeleton.

\subsubsection{Biological Evaluation}

Antifungal Activity. Antifungal activities of some complexes were studied against one fungal strain "Sclerotium rolfsi." It is concluded that all the synthesized complexes showed overall good activity against this antifungal strain up to 90\% (Figures 6(a), 6(b), 6(c), and 6(d)). From the results found (Table 7 ), it has been concluded that, on increasing the concentration of the complexes, the colony diameter of the fungus decreases and hence percent inhibition increases. On doubling the concentration of the complexes, the percent inhibition also doubles, which shows linear relationship between concentration and percent inhibition. The increase in biological activity is due to faster diffusion of metal complexes as a whole through the cell membrane or due to 
TABLE 7: In vitro efficacy of axially ligated $\mathrm{X}-\mathrm{Zn}-\mathrm{t}\left(p-\mathrm{CH}_{3}\right) \mathrm{PP}(\mathrm{X}=$ different phenols as axial ligand $)$ against the pathogen Sclerotium rolfsi. Colony diameter of control $C=90 \mathrm{~mm}$.

\begin{tabular}{|c|c|c|c|}
\hline Porphyrins & $\begin{array}{l}\text { Different concentration } \\
\text { (ppm) of the complexes }\end{array}$ & $\begin{array}{l}\text { Colony diameter } \\
\text { (in } \mathrm{mm} \text { ) at different } \\
\text { concentrations }\end{array}$ & $\begin{array}{c}\% \text { inhibition } \\
I=[(C-T) / C] * 100 \text { at } \\
\text { different concentrations } \\
(\mathrm{ppm})\end{array}$ \\
\hline \multirow{3}{*}{$\begin{array}{l}p-\mathrm{NH}_{2} \mathrm{phO}-\mathrm{Zn}-\mathrm{t}\left(p-\mathrm{CH}_{3}\right) \mathrm{PP} \\
{\left[\left(\mathrm{C}_{6} \mathrm{H}_{6} \mathrm{NO}\right) \mathrm{Zn}\left(\mathrm{C}_{48} \mathrm{H}_{36} \mathrm{~N}_{4}\right)\right]}\end{array}$} & 100 & 52.62 & 41.53 \\
\hline & 200 & 38.87 & 56.81 \\
\hline & 300 & 28 & 68.88 \\
\hline \multirow{3}{*}{$\begin{array}{l}p-\mathrm{CH}_{3} \mathrm{phO}-\mathrm{Zn}-\mathrm{t}\left(p-\mathrm{CH}_{3}\right) \mathrm{PP} \\
{\left[\left(\mathrm{C}_{7} \mathrm{H}_{7} \mathrm{O}\right) \mathrm{Zn}\left(\mathrm{C}_{48} \mathrm{H}_{36} \mathrm{~N}_{4}\right)\right]}\end{array}$} & 100 & 49.12 & 45.4 \\
\hline & 200 & 40.25 & 55.27 \\
\hline & 300 & 31.3 & 65.22 \\
\hline \multirow{3}{*}{$\begin{array}{l}p-\mathrm{OCH}_{3} \mathrm{phO}-\mathrm{Zn}-\mathrm{t}\left(p-\mathrm{CH}_{3}\right) \mathrm{PP} \\
{\left[\left(\mathrm{C}_{7} \mathrm{H}_{7} \mathrm{O}_{2}\right) \mathrm{Zn}\left(\mathrm{C}_{48} \mathrm{H}_{36} \mathrm{~N}_{4}\right)\right]}\end{array}$} & 100 & 51 & 43.33 \\
\hline & 200 & 21.87 & 75.69 \\
\hline & 300 & 11.62 & 87.08 \\
\hline \multirow{3}{*}{$\begin{array}{l}m-\mathrm{NO}_{2} \mathrm{phO}-\mathrm{Zn}-\mathrm{t}\left(p-\mathrm{CH}_{3}\right) \mathrm{PP} \\
{\left[\left(\mathrm{C}_{6} \mathrm{H}_{4} \mathrm{NO}_{3}\right) \mathrm{Zn}\left(\mathrm{C}_{48} \mathrm{H}_{36} \mathrm{~N}_{4}\right)\right]}\end{array}$} & 100 & 48 & 46.66 \\
\hline & 200 & 36.5 & 59.44 \\
\hline & 300 & 25.5 & 71.66 \\
\hline \multirow{3}{*}{$\begin{array}{l}p \text {-ClphO-Zn-t }\left(p-\mathrm{CH}_{3}\right) \mathrm{PP} \\
{\left[\left(\mathrm{C}_{6} \mathrm{H}_{4} \mathrm{ClO}\right) \mathrm{Zn}\left(\mathrm{C}_{48} \mathrm{H}_{36} \mathrm{~N}_{4}\right)\right]}\end{array}$} & 100 & 37.5 & 58.33 \\
\hline & 200 & 21.12 & 76.52 \\
\hline & 300 & 12.75 & 85 \\
\hline
\end{tabular}

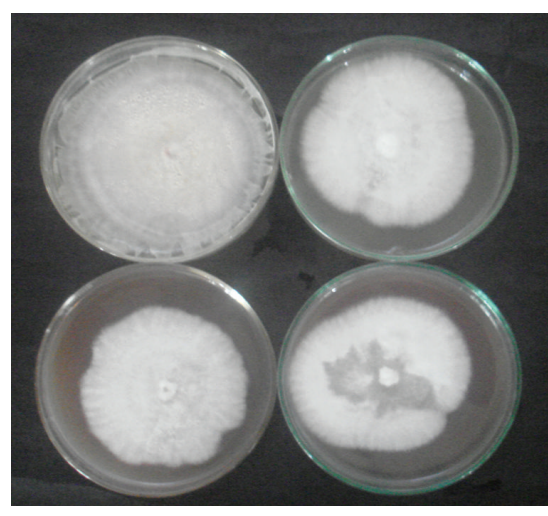

(a)

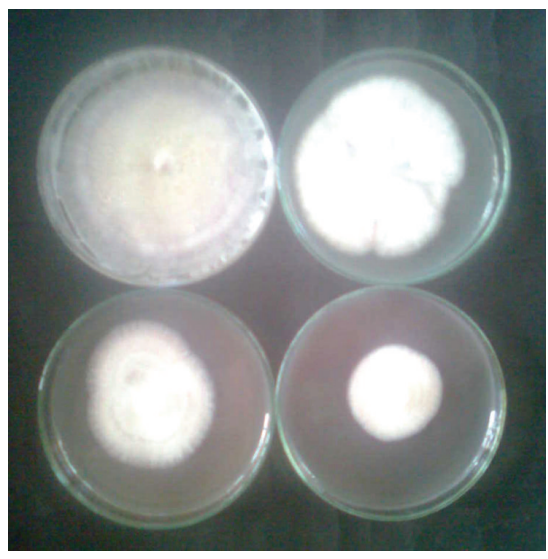

(c)

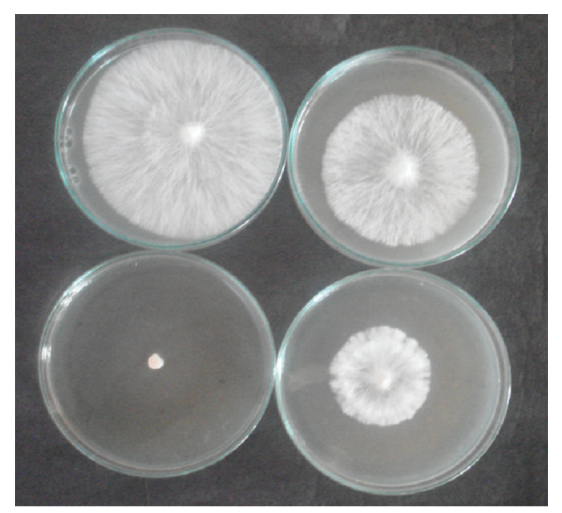

(b)

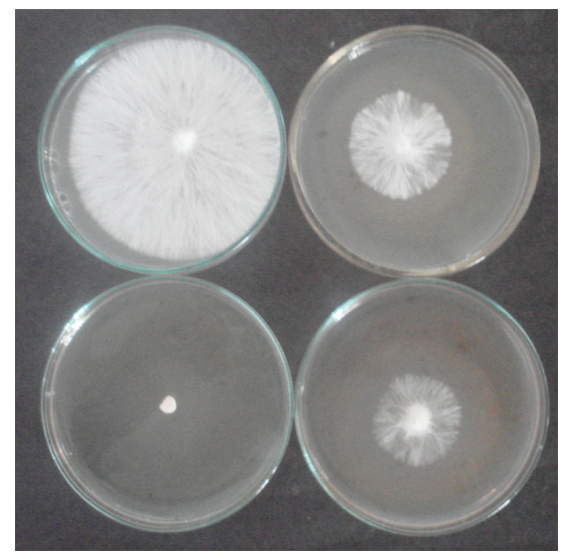

(d)

Figure 6: (a) Antifungal activity of $p-\mathrm{NH}_{2}$ phO- $\mathrm{Zn}-\mathrm{t}\left(p-\mathrm{CH}_{3}\right) \mathrm{PP}$, (b) antifungal activity of $p-\mathrm{OCH}_{3}$ phO-Zn-t $\left(p-\mathrm{CH}_{3}\right) \mathrm{PP},(\mathrm{c})$ antifungal activity of $p-\mathrm{NO}_{2}$ phO-Zn-t $\left(p-\mathrm{CH}_{3}\right) \mathrm{PP}$, and (d) antifungal activity of $p-\mathrm{ClphO}-\mathrm{Zn}-\mathrm{t}\left(p-\mathrm{CH}_{3}\right) \mathrm{PP}$. 


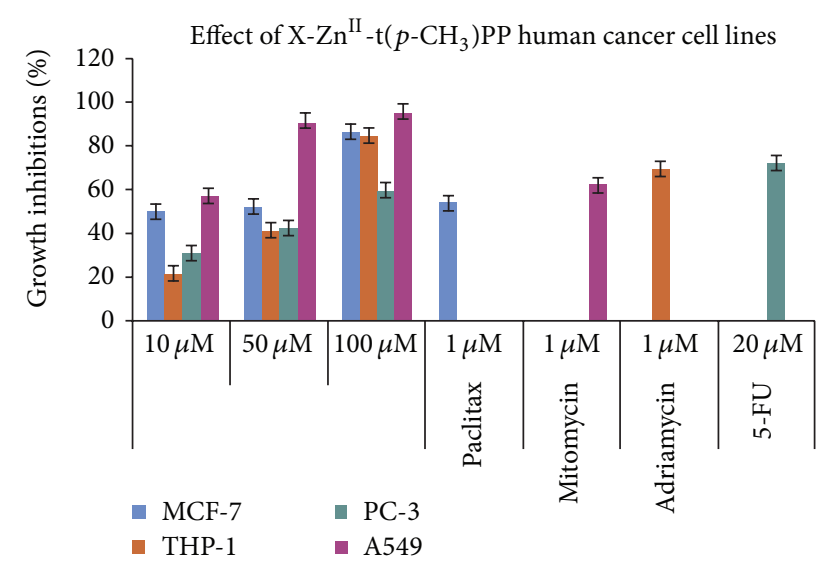

FIGURE 7: In vitro cytotoxicity of $p-\mathrm{NO}_{2}$ phO-Zn-t $\left(p-\mathrm{CH}_{3}\right) \mathrm{PP}$ complexes against human cancer cell lines.

combined effect of metal atom and the ligand. Such increased activity of the metal complexes can be explained on the basis of Overtone's concept [46] and Tweedy's chelation theory [47]. The lipid membrane that surrounds the cell favors the passage of only lipid soluble materials, due to liphophilicity being an important factor which controls the antimicrobial activity. On chelation, the polarity of the metal ion will be reduced to a greater extent due to overlap of the ligand orbital and partial sharing of the positive charge of the metal ion with donor group.

In Vitro Cytotoxicity. Evaluation of in vitro cytotoxicity of the corresponding ligand was also observed against four human cancer cell lines, namely, Breast (MCF-7), Leukemia (THP-1), Prostate (PC-3), and Lung (A549), at different concentrations by using SRB assay as shown in Figure 7. Dose dependent percent growth inhibition was observed against all the cancer cell lines. Among the substituted oxygen donor ligands, $p$ $\mathrm{NO}_{2}$ phO-Zn-t $\left(p-\mathrm{CH}_{3}\right) \mathrm{PP}$ showed prominent activity against three A549, MCF-7, and THP-1 human cancer cell lines. Highest growth percent inhibition was observed against Lung cancer cell line and lowest percent growth inhibition was observed against Prostate cancer cell line by the ligand. The percent growth inhibition observed for the ligand was 57, 90, and 95 against Lung, 50, 52, and 86 against Breast, and 21, 41, and 84 against leukemia cancer cell lines at 10,50 , and $100 \mu \mathrm{M}$, respectively, because the presence of electron-withdrawing nitro group $\left(-\mathrm{NO}_{2}\right)$ on the phenolic ring in general increases the antimicrobial activities of the tested metal complexes compared to complexes having no substituent. These results suggested that metal complexes had effective improvement of bioavailability, and electron-withdrawing nitro group had effective and direct impact on selective anticancer activities. Hence, therefore, the ligand $p-\mathrm{NO}_{2}$ phO, which is axially ligated with $\mathrm{Zn}-\mathrm{t}\left(p-\mathrm{CH}_{3}\right) \mathrm{PP}$, shows overall better activity than its free base $\mathrm{H}_{2}-\mathrm{t}\left(p-\mathrm{CH}_{3}\right) \mathrm{PP}$ and metallated $\mathrm{Zn}-\mathrm{t}(p-$ $\left.\mathrm{CH}_{3}\right)$ PP. The complex $p-\mathrm{NO}_{2}$ phO-Zn-t $\left(p-\mathrm{CH}_{3}\right) \mathrm{PP}$ showed less than 59\% growth inhibition against Prostate (PC-3) human cancer cell line.

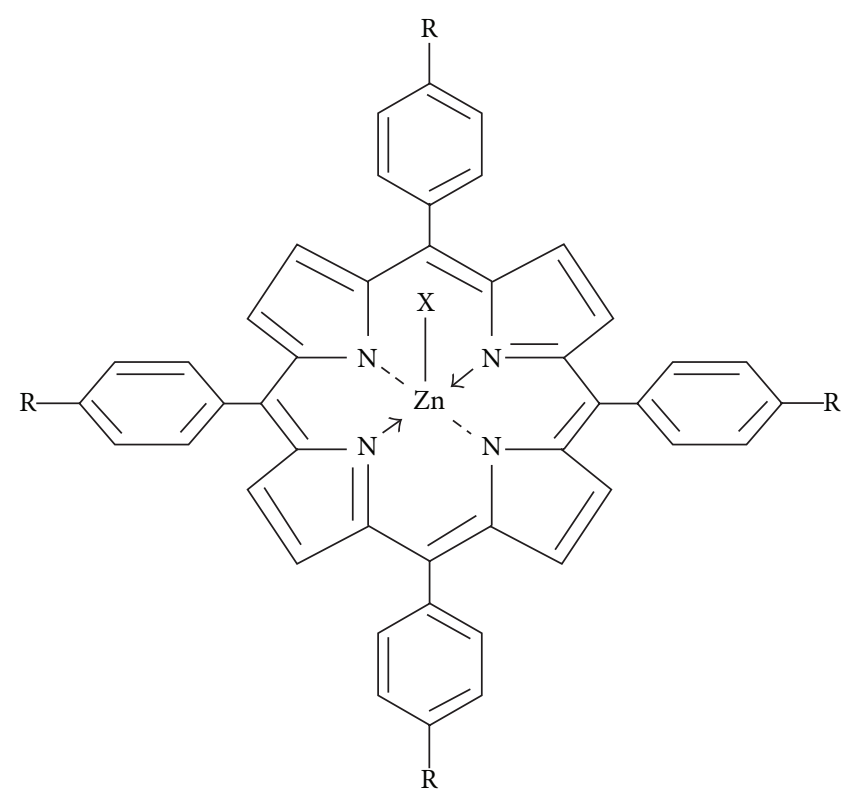

FIgURE 8: Proposed structure of axially ligated zinc(II) porphyrins.

\section{Conclusion}

On the basis of physicochemical and spectral evidences it is found that all the complexes with a general formula X-Zn-t $(p-$ $\left.\mathrm{CH}_{3}\right) \mathrm{PP}(\mathrm{X}=$ different phenolates as axial ligand $)$ in which the four-coordinate zinc porphyrin will accept one and only one axial ligand in 1:1 molar ratio to form five-coordinated complexes. The proposed structure for the complexes under investigation with general formula $\left[\mathrm{X}-\mathrm{Zn}-\mathrm{t}\left(p-\mathrm{CH}_{3}\right) \mathrm{PP}\right]$ is given as Figure 8. Also, biological evaluation (antifungal and anticancer activities) of the synthesized complexes shows that these complexes have potential against fungal growth. Moreover, for anticancer activity, highest growth percent inhibition was observed against Lung cancer cell line and lowest percent growth inhibition was observed against Prostate cancer cell line by the ligand.

\section{Conflict of Interests}

The authors declare that they have no conflict of interests.

\section{References}

[1] V. K. Gupta, D. K. Chauhan, V. K. Saini, S. Agarwal, M. M. Antonijevic, and H. Lang, "A porphyrin based potentiometric sensor for $\mathrm{Zn}^{2+}$ determination," Sensors, vol. 3, no. 7, pp. 223235, 2003.

[2] C.-T. Chen, "Evolution of red organic light-emitting diodes: materials and devices," Chemistry of Materials, vol. 16, no. 23, pp. 4389-4400, 2004.

[3] O. S. Wolfbeis, "Fiber-optic chemical sensors and biosensors," Analytical Chemistry, vol. 78, no. 12, pp. 3859-3873, 2006.

[4] V. K. Gupta, S. Chandra, D. K. Chauhan, and R. Mangla, "Membranes of 5,10,15,20-tetrakis(4-methoxyphenyl) porphyrinatocobalt (TMOPP-Co) (I) as $\mathrm{MoO}_{4}^{2-}$-selective sensors," Sensors, vol. 2, no. 5, pp. 164-173, 2002. 
[5] K. I. Ozoemena, "Anodic oxidation and amperometric sensing of hydrazine at a glassy carbon electrode modified with cobalt (II) phthalocyanine-cobalt (II) tetraphenylporphyrin (CoPc(CoTPP)4) supramolecular complex," Sensors, vol. 6, no. 8, pp. 874-891, 2006.

[6] O. Ikeda, K. Yoshinaga, and J. Lei, "Nitric oxide detection with glassy carbon electrodes coated with charge-different polymer films," Sensors, vol. 5, no. 4-5, pp. 161-170, 2005.

[7] R. Purrello, A. Raudino, L. Monsù Scolaro, A. Loisi, E. Bellacchio, and R. Lauceri, "Ternary porphyrin aggregates and their chiral memory," Journal of Physical Chemistry B, vol. 104, no. 46, pp. 10900-10908, 2000.

[8] H. S. Cho, D. H. Jeong, S. Cho et al., "Photophysical properties of porphyrin tapes," Journal of the American Chemical Society, vol. 124, no. 49, pp. 14642-14654, 2002.

[9] J. Yu, X. Wang, B. Zhang, Y. Weng, and L. Zhang, "Prolonged excited-state lifetime of porphyrin due to the addition of colloidal $\mathrm{SiO}_{2}$ to Triton X-100 micelles," Langmuir, vol. 20, no. 5, pp. 1582-1586, 2004.

[10] S. Tangestaninejad, M. H. Habibi, V. Mirkhani, and M. Moghadam, "Mn ( $\mathrm{Br}_{8}$ TPPS) supported on Amberlite IRA-400 as a robust and efficient catalyst for alkene epoxidation and alkane hydroxylation," Molecules, vol. 7, no. 2, pp. 264-270, 2002.

[11] E. G. Girichev, M. I. Bazanov, N. Z. Mamardashvili, and A. Gjeyzak, "Electrochemical and electrocatalytical properties of 3,7,13,17-tetramethyl- 2,8,12,18-tetrabutylporphyrin in alkaline solution," Molecules, vol. 5, no. 6, pp. 767-774, 2000.

[12] B. Gao, Y. Chen, and Q. Lei, "Hydroxylationof cyclohexanewith molecular oxygen catalyzed by highly efficient heterogeneous $\mathrm{Mn}$ (III) porphyrin catalysts prepared by special synthesis and immobilization method," Journal of Inclusion Phenomena and Macrocyclic Chemistry, vol. 74, no. 1-4, pp. 455-465, 2012.

[13] A. E. O’Connor, W. M. Gallagher, and A. T. Byrne, "Porphyrin and nonporphyrin photosensitizers in oncology: preclinical and clinical advances in photodynamic therapy," Photochemistry and Photobiology, vol. 85, no. 5, pp. 1053-1074, 2009.

[14] M. Wathier and M. W. Grinstaff, "Synthesis and properties of supramolecular ionic networks," Journal of the American Chemical Society, vol. 130, no. 30, pp. 9648-9649, 2008.

[15] C. M. Drain, A. Varotto, and I. Radivojevic, "Self-organized porphyrinic materials," Chemical Reviews, vol. 109, no. 5, pp. 16301658, 2009.

[16] D. Vlascici, E. F. Cosma, E. M. Pica et al., "Free base porphyrins as ionophores for heavy metal sensors," Sensors, vol. 8, no. 8, pp. 4995-5004, 2008.

[17] D. Aviezer, S. Cotton, M. David et al., "Porphyrin analogues as novel antagonists of fibroblast growth factor and vascular endothelial growth factor receptor binding that inhibit endothelial cell proliferation, tumor progression, and metastasis," Cancer Research, vol. 60, no. 11, pp. 2973-2980, 2000.

[18] H. Imahori, Y. Mori, and Y. Matano, "Nanostructured artificial photosynthesis," Journal of Photochemistry and Photobiology C, vol. 4, no. 1, pp. 51-83, 2003.

[19] A. Forneli, M. Planells, M. A. Sarmentero et al., "The role of para-alkyl substituents on meso-phenyl porphyrin sensitised $\mathrm{TiO}_{2}$ solar cells: control of the $\mathrm{e}_{\mathrm{TiO} 2}$ /electrolyte ${ }^{+}$recombination reaction," Journal of Materials Chemistry, vol. 18, no. 14, pp. 1652-1658, 2008.
[20] A. D. Adler, F. R. Longo, J. D. Finarelli, J. Goldmacher, J. Assour, and L. Korsakoff, "A simplified synthesis for mesotetraphenylporphin," Journal of Organic Chemistry, vol. 32, no. 2, p. 476, 1967.

[21] K. M. Kadish, K. M. Smith, and R. Guilard, The Porphyrin Handbook, Academic Press, San Diego, Calif, USA, 2000.

[22] K. Saito, Y. Kashiwagi, K. Ohkubo, and S. Fukuzumi, "An extremely long-lived charge-separated state of zinc tetraphenylporphyrin coordinated with pyridylnaphthalene-diimide," Journal of Porphyrins and Phthalocyanines, vol. 10, no. 12, pp. 13711379, 2006.

[23] V. K. K. Praneeth, F. Paulat, T. C. Berto et al., "Electronic structure of six-coordinate iron(III)-porphyrin NO adducts: the elusive iron(III)-NO(radical) state and its influence on the properties of these complexes," Journal of the American Chemical Society, vol. 130, no. 46, pp. 15288-15303, 2008.

[24] A. S. Semeikin, O. I. Koifman, and B. D. Berezin, "Synthesis of tetraphenylporphins with active groups in the phenyl rings. 1. Preparation of tetrakis(4-aminophenyl)porphin," Chemistry of Heterocyclic Compounds, vol. 18, no. 10, pp. 1046-1047, 1982.

[25] K. M. Kadish, K. M. Smith, and R. Guilard, "Biochemistry and binding activation of small molecules," in The Porphyrin Handbook, Academic Press, New York, NY, USA, 1999.

[26] M. Nappa and J. S. Valentine, "The influence of axial ligands on metalloporphyrin visible absorption spectra. Complexes of tetraphenylporphinatozinc," Journal of the American Chemical Society, vol. 100, no. 16, pp. 5075-5080, 1978.

[27] M.-Y. R. Wang and B. M. Hoffman, "Systematic trends in metalloporphyrin optical spectra," Journal of the American Chemical Society, vol. 106, no. 15, pp. 4235-4240, 1984.

[28] I. Stojiljkovic, B. D. Evavold, and V. Kumar, "Antimicrobial properties of porphyrins," Expert Opinion on Investigational Drugs, vol. 10, no. 2, pp. 309-320, 2001.

[29] K. Rajesh, A. K. Rahiman, K. S. Bharathi, S. Sreedaran, V. Gangadevi, and V. Narayanan, "Spectroscopic, redox and biological studies of push-pull porphyrins and their metal complees," Bulletin of the Korean Chemical Society, vol. 31, no. 9, pp. 26562664, 2010.

[30] J. Bozja, J. Sherrill, S. Michielsen, and I. Stojiljkovic, "Porphyrinbased, light-activated antimicrobial materials," Journal of Polymer Science, Part A, vol. 41, no. 15, pp. 2297-2303, 2003.

[31] R. A. Sanchez-Delgado, K. Lazardi, L. Rincon, and J. A. Urbina, "Toward a novel metal-based chemotherapy against tropical diseases. 1. Enhancement of the efficacy of clotrimazole against Trypanosoma cruzi by complexation to ruthenium in $\mathrm{RuCl}_{2}$ (clotrimazole) $)_{2}$, Journal of Medicinal Chemistry, vol. 36, pp. 2041-2043, 1993.

[32] M. T. H. Tarafder, K. T. Jin, K. A. Crouse, A. M. Ali, B. M. Yamin, and H.-K. Fun, "Coordination chemistry and bioactivity of $\mathrm{Ni}^{2+}, \mathrm{Cu}^{2+}, \mathrm{Cd}^{2+}$ and $\mathrm{Zn}^{2+}$ complexes containing bidentate schiff bases derived from S-benzyldithiocarbazate and the X-ray crystal structure of bis[S-benzyl- $\beta-N$-(5-methyl-2furylmethylene)dithiocarbazato]cadmium(II)," Polyhedron, vol. 21, no. 25-26, pp. 2547-2554, 2002.

[33] J. Sheikh, H. Juneja, V. Ingle, P. Ali, and T. B. Hadda, "Synthesis and in vitro biology of $\mathrm{Co}(\mathrm{II}), \mathrm{Ni}(\mathrm{II}), \mathrm{Cu}(\mathrm{II})$ and $\mathrm{Zinc}(\mathrm{II}) \mathrm{com}$ plexes of functionalized beta-diketone bearing energy buried potential antibacterial and antiviral O,O pharmacophore sites," Journal of Saudi Chemical Society, vol. 17, pp. 269-276, 2013.

[34] J. R. Platt and H. B. Klevens, "Spectroscopy of organic molecules in the vacuum ultraviolet," Reviews of Modern Physics, vol. 16, no. 3-4, pp. 182-223, 1944. 
[35] G. P. Gurinovich, A. N. Sevchenko, and K. N. Solovyev, "Spectroscopy of chlorophyll and alliedcompounds," Nauka $i$ Tekhnika. In press.

[36] L. A. Nafie, M. Pézolet, and W. L. Peticolas, "On the origin of the intensity of the resonant raman bands of differing polarization in heme proteins," Chemical Physics Letters, vol. 20, no. 6, pp. 563-568, 1973.

[37] J. M. Vincent, "Distortion of fungal hyphæ in the presence of certain inhibitors," Nature, vol. 159, no. 4051, p. 850, 1947.

[38] A. Thiantanawat, B. J. Long, and A. M. Brodie, "Signaling pathways of apoptosis activated by aromatase inhibitors and antiestrogens," Cancer Research, vol. 63, no. 22, pp. 8037-8050, 2003.

[39] X. Tong, S. Lin, M. Fujii, and D.-X. Hou, "Echinocystic acid induces apoptosis in HL-60 cells through mitochondriamediated death pathway," Cancer Letters, vol. 212, no. 1, pp. 2132, 2004.

[40] H. Scheer and J. J. Katz, "Nuclear magnetic resonance spectroscopy of porphyrins and metalloporphyrins," in Porphyrins and Metalloporphyrins, K. M. Smith, Ed., p. 399, Elsevier, Amsterdam, The Netherlands, 1975.

[41] E. Fagadar-Cosma, C. Enache, I. Armeanu et al., "The influence of $\mathrm{pH}$ over topography and spectroscopic properties of silica hybrid materials embedding meso-tetratolylporphyrin," Materials Research Bulletin, vol. 44, no. 2, pp. 426-431, 2009.

[42] K. Schweiger, M. Goldner, H. Huckstadt, and H. Z. Homborg, "Synthese and Eigen Schaften Von cis Diacidophthalocyaninato(2-)thallaton(III); kristallstruktur von Tetra(n-butyl)ammonium-cisdinitro $\left(\mathrm{O}, \mathrm{O}^{\prime}\right)$-and-cis-dichlorophthalocyaninato(2-)thallat(III)," Zeitschrift für Anorganische und Allgemeine Chemie, vol. 625, no. 10, pp. 1693-1699, 1999.

[43] R. C. Dougherty, in Biochemical Applications ofMass Spectrometry, G. R. Waller, Ed., vol. 6, p. 1288, John Wiley \& Sons, New York, NY, USA, 1972.

[44] C. Timiriazeff, "Colourless chlorophyll," Nature, vol. 32, no. 824, p. $342,1885$.

[45] J.-P. Strachan, S. Gentemann, J. Seth et al., "Effects of orbital ordering on electronic communication in multiporphyrin arrays," Journal of the American Chemical Society, vol. 119, no. 46, pp. 11191-11201, 1997.

[46] N. Dharmaraj, P. Viswanathamurthi, and K. Natarajan, "Ruthenium(II) complexes containing bidentate Schiff bases and their antifungal activity," Transition Metal Chemistry, vol. 26, no. 1-2, pp. 105-109, 2001.

[47] L. Mishra and V. K. Singh, "Co(ll), Ni(ll) and $\mathrm{Cu}(\mathrm{II})$ and $\mathrm{Zn}(\mathrm{II})$ complexes with Schiff bases derived from 2-aminobenzimidazoles and pyrazolycarboxaldehyde," Indian Journal of Chemistry, vol. 32, p. 446, 1993. 

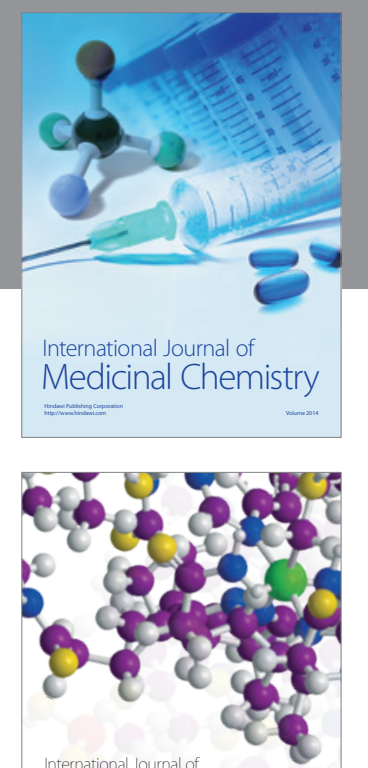

\section{Carbohydrate} Chemistry

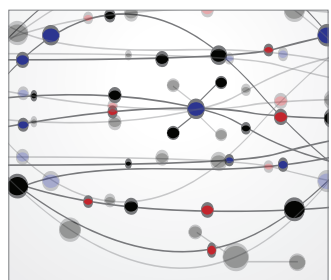

The Scientific World Journal
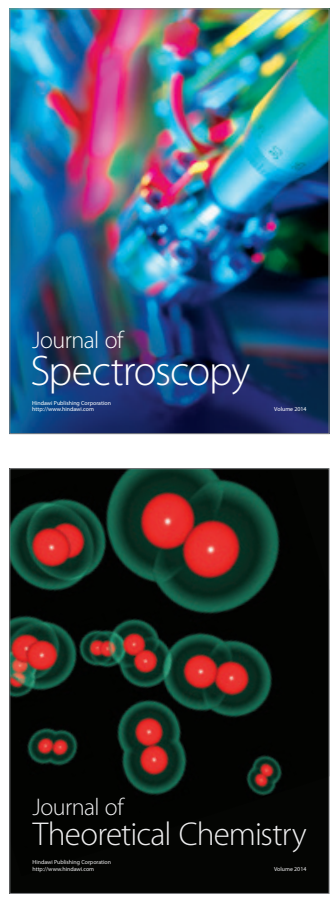
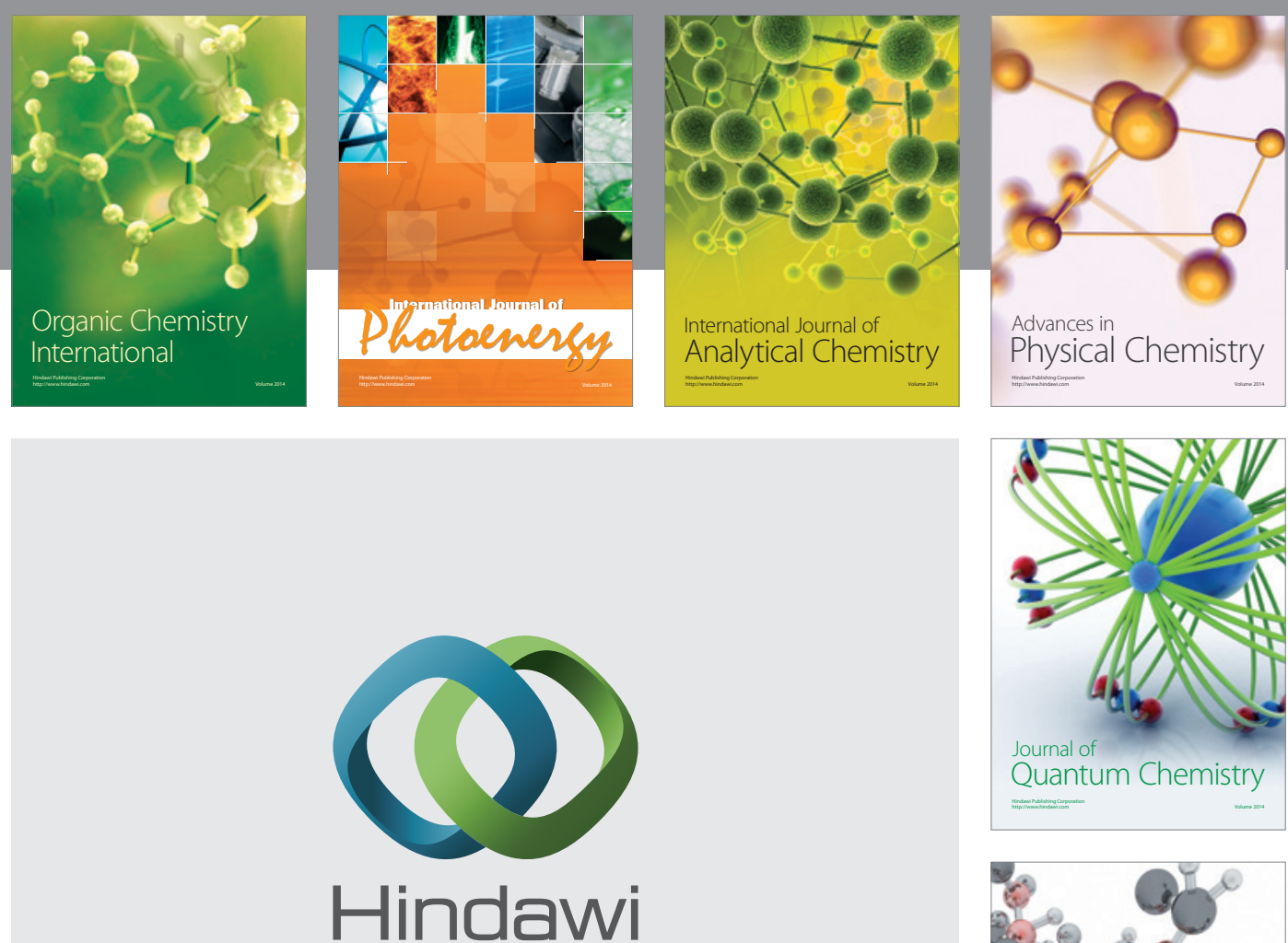

Submit your manuscripts at

http://www.hindawi.com

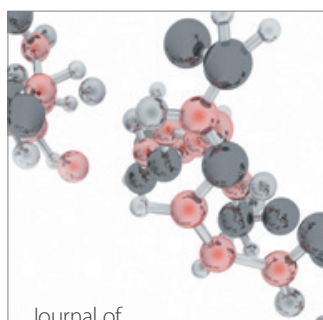

Analytical Methods

in Chemistry

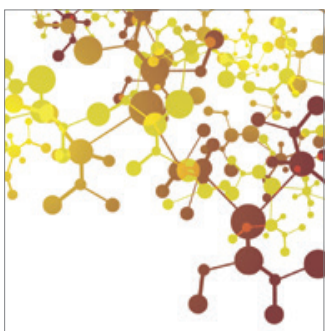

Journal of

Applied Chemistry

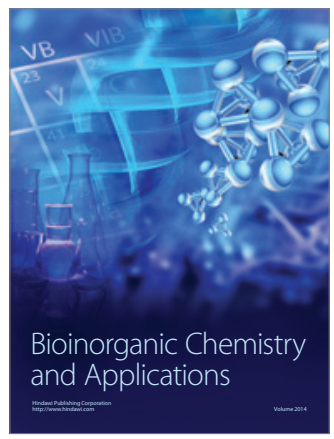

Inorganic Chemistry
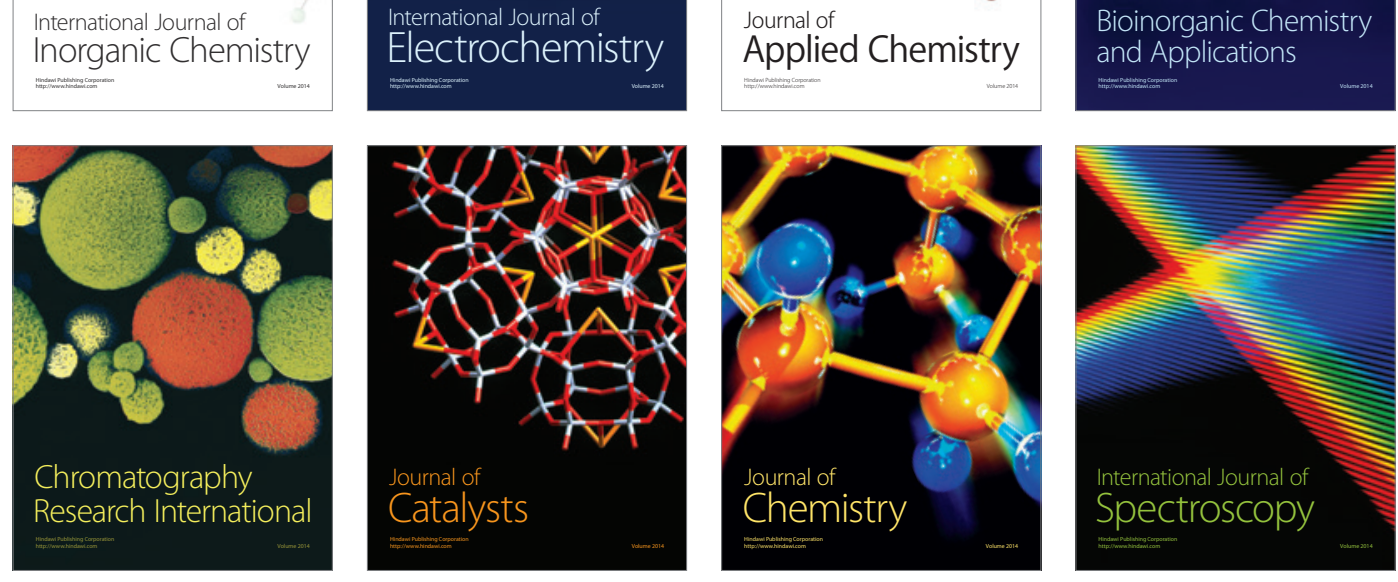